Pacific Journal of Mathematics

ON REPRESENTATIONS OF DISCRETE, FINITELY
GENERATED, TORSION-FREE, NIL POTENT GROUPS 


\title{
ON REPRESENTATIONS OF DISCRETE, FINITELY GENERATED, TORSION-FREE, NILPOTENT GROUPS
}

\author{
ROGER E. HOWE
}

\begin{abstract}
With A. A. Kirillov's work on the representations of nilpotent lie groups, a new chapter in the theory of group representations opened. Subsequent papers of Bernat, Moore and Auslander-Kostant have further demonstrated the power of the methods introduced by Kirillov. The purpose of this paper is to begin an extension of these methods in yet another direction. Specifically, the object here is to calculate the primitive ideal spaces of the groups indicated in the title.
\end{abstract}

While this again is a fairly special extension of what eventually should be a very far-reaching theory, it has present merit for several reasons: (1) Much work necessary for further extensions is already done in handling these particular groups. In particular, slight modifications and extensions of these methods allow one to deal with most unipotent groups that occur in arithmetic; (2) Most of these groups are not type I (see Thoma [14]); hence they provide examples of what can be said about such "bad" groups; (3) The theory of these groups also sheds light on the harmonic analysis of finite $p$-groups.

From now on, $\Gamma$ will always denote a discrete, finitely generated, torsion-free, nilpotent group. $e$ will be its identity element, $\Gamma=\Gamma^{(1)}$, $\Gamma^{(2)}, \Gamma^{(3)}$, etc. its descending central series, and $\mathscr{Z}(\Gamma)$, $\mathscr{L}^{(2)}(\Gamma)$, etc. its ascending central series. If $\Gamma^{(k+1)}=\{e\}$, we will say $\Gamma$ is $k$-step nilpotent.

To carry out for $\Gamma$ a program analogous to Kirillov's we need for $\Gamma$ a dual of a lie algebra, and so, first, a lie algebra. Malcev (see [1]) has shown that any $\Gamma$ such as we are considering may be embedded as a discrete subgroup in a simply connected nilpotent lie group $\mathscr{N}$, so that the quotient $\mathscr{N} / \Gamma$ is compact; furthermore $\mathscr{N}$ and the embedding are unique up to isomorphism. From now on, $\mathscr{N}$ will always denote this group, and we will consider $\Gamma$ to be a subgroup of $\mathscr{N}$ whenever this is convenient.

$N$ will denote the lie algebra of $\mathscr{N}$. Then exp: $N \rightarrow \mathscr{N}$ is a diffeomorphism, with $\log$ as its inverse. We would like to say $\log \Gamma \leqq N$ is the lie algebra of $\Gamma$. However, this makes no sense in general, because $\log \Gamma$ need be closed neither under addition, nor under taking commutators. But suppose $\mathscr{N}$ is $k$-step nilpotent, and $L \subseteq N$ is a lattice, such that $[L, L] \subseteq k ! L$. Then an easy calculation with the Campbell-Hausdorff formula ([13]) shows that $\Gamma=$ 
$\exp L$ is a subgroup of $\mathscr{N}$. We will call such a lattice $L$, and $\exp L$, elementarily exponentiable, or e.e., for short.

Then given an e.e. group $\Gamma$, it has a well defined lie algebra $L=$ $\log \Gamma$, and we may consider $\hat{L}$, the dual group to $L$. Via exp, any $\psi \in \hat{L}$ may be considered to be a function on $\Gamma$; and will be whenever convenient. Now inner automorphisms of $\Gamma$ induce an action of $\Gamma$ on $L$ by automorphisms, denoted by Ad, and by duality, an action $\mathrm{Ad}^{*}$ on $\hat{L}$. More generally, if $\Gamma$ is not necessarily e.e., but $\Gamma_{1} \subseteq \Gamma$ is e.e. and normal, $\Gamma$ acts on $\hat{L}_{1}=\widehat{\log \Gamma_{1}}$ by $\mathrm{Ad}^{*}$. We will denote the quasiorbit space ([5]) of this action by $\Delta\left(\Gamma_{1}, \Gamma\right)$.

To avoid problems in ergodic theory, we will not attempt to compute representations of $\Gamma$, but only primitive ideals of $C^{*}(\Gamma)$, the enveloping $C^{*}$-algebra of $l_{1}(\Gamma)$ (see [6] and [12]). We denote the primitive ideal space of a group $G$, with the hull-kernel topology, by $M(G)$. More generally if $H \subseteq G$ is normal, we consider the relative primitive ideal space, $M(H, G)$. This is defined as follows. $G$ acts on $M(H)$ by conjugation. As $H$ is in the kernel of this action, we denote this action by $\mathrm{Ad}^{*} G / H$. Then $M(H, G)$ is defined as the quasiorbit space of $\mathrm{Ad}^{*} G / H$ acting on $M(H)$. As we will see later, $M(H, G)$ also has an interpretation in terms of restrictions to $H$ of irreducible representations of $G$. The main theorem of this paper may now be stated as follows:

THEOREM. Let $\Gamma$ be k-step nilpotent and e.e., $L=\log \Gamma, L^{\prime}=$ $2 L, \quad \Gamma^{\prime}=\exp L^{\prime}$. Then there is a canonical homeomorphism $\alpha: \Delta\left(\Gamma^{\prime}, \Gamma\right) \rightarrow M\left(\Gamma^{\prime}, \Gamma\right)$.

To arrive at this theorem, we have had to restrict our attention to e.e. groups. It is therefore of interest to know how general they are. In this regard, we have

Proposition 0. If $\Gamma$ is k-step nilpotent, but not e.e., let $\log \Gamma^{* *}$ denote the lattice generated in $N$ by $\log \Gamma$. Then $k !^{2} \log \Gamma^{* *} \subseteq$ $\log \Gamma$, and $\left[\log \Gamma^{* *}, \log \Gamma^{* *}\right] \subseteq 1 /(k-1) ! \log \Gamma^{* *}$. Hence there exist e.e. lattices $L_{1}, L_{2}$ such that $L_{1} \subseteq \log \Gamma \subseteq L_{2}$, and $k !^{2} \log \Gamma^{* *} \subseteq L_{1}$, and $(k !(k-1) !)^{k-1} \quad L_{2} \cong \log \Gamma^{* *}$.

If $\Gamma_{1} \subseteq \Gamma$ is e.e., and of finite index, then a very slight variation of the theorem allows computation of $M\left(\Gamma_{1}, \Gamma\right)$ and as we shall see there is a natural surjective map $r: M(\Gamma) \rightarrow M\left(\Gamma_{1}, \Gamma\right)$ which is boundedly finitely many to one. Thus, up to a finite covering, one can calculate $M(\Gamma)$ for any $\Gamma$.

Here is how the paper is organized. In §II we analyze the finite dimensional representations of an e.e. $\Gamma$. Roughly speaking, 
they correspond to finite $\mathrm{Ad}^{*} \Gamma$ orbits, and in a sense, form the "rational" points of $M(\Gamma)$. They are dense in $M(\Gamma)$. We obtain character formulas, from which multiplicities of induced representations and tensor products may be read off. In $\S$ III, we develop some machinery which facilitates calculation of primitive ideal spaces. In $\S I V$, the structure of ideals of $C^{*}(\Gamma)$ is investigated. We show that to each primitive ideal, a trace may be associated in a unique and nice way. In $\S \mathrm{V}$, the structure of $\mathrm{Ad}^{*} \Gamma$ orbits is analyzed in a fashion as parallel as possible to that of $\S I V$, and the main result is established. In $\S \mathrm{VI}$, we apply some of the results to harmonic analysis of finite $p$-groups. In an appendix, we prove Proposition 0 . Throughout, we lean very heavily on the Campbell-Hausdorff formula, which we abbreviate C. H. Results of calculations using it will simply be stated.

Perhaps, before beginning, it would be instructive to provide an example of a particular primitive ideal space. Let $H$ be the group of upper triangular integral unipotent $3 \times 3$ matrices; i.e., the "discrete Heisenberg group." Then $\mathscr{\not}(H) \cong Z$, and there is a natural fibration $\pi: M(H) \rightarrow T=\hat{Z}$. The fiber over a torsion element of $T$ is a two-torus, and over an irrational, or nontorsion, element, is a single point. The topology of $M(H)$ is such that any set-theoretic section of $\pi$ is continuous.

I should like here to express my thanks to Calvin Moore. The contents of this paper are essentially my doctoral dissertation, done under his guidance. His influence on my development and direction has been both beneficial and far-reaching.

II. Finite dimensional representations of $\Gamma$. We begin with some definitions and notation. Let $G$ be a group. If $a, b \in G$, then $(a, b)=a b a^{-1} b^{-1}$ is the commutator of $a$ and $b$. If $A, B \leqq G$, then $(A, B)=\{(a, b), a \in A, b \in B\}$. If $H \subseteq G$ is a subgroup and $V$ is a representation of $H$, then $U^{V, H}$ or $U^{V}$ is the corresponding induced representation of $G$. If $V$ has a character $\psi$, then $U^{\psi, H}$ or $U^{\psi}$ may also be used.

If again $H \cong G$ is a subgroup, we say $H$ is saturated if $g^{n} \in H$ implies $g \in H$, for any $g \in G$. Given $H, H_{s}$ will denote the smallest saturated subgroup containing $H$. Evidently, if $H$ is normal, $H$ is saturated if and only if $G / H$ is torsion-free.

If $\Gamma^{\prime} \subseteq \Gamma$, then $\Gamma^{\prime}$ is of finite index in $\Gamma_{s}^{\prime}$. In terms of $\mathscr{N}$ and $N, \log \Gamma_{s}^{\prime}=\log \Gamma \cap N^{\prime}$, where $N^{\prime}$ is the real subspace of $N$ spanned by $\log \Gamma^{\prime}$.

If $X$ is any set, $f$ any function on $X$, and $Y \cong X$ any subset, then $f_{\mid Y}$ is the restriction of $f$ to $Y$. If $f$ is a complex-valued function on $Y, \dot{f}$ is the extension of $f$ to $X$, which is identically zero 
off $Y$. If $A$ is a finite set, $\#(A)$ will denote the cardinality of $A$.

Now, given $\Gamma$, the family of groups $\left\{\exp \left(n k !^{2} \log \Gamma^{* *}\right)\right\}$ form a family of e.e. characteristic subgroups of $\Gamma$ of finite index, such that any subgroup of finite index contains one of them, and their total intersection reduces to the identity. This implies that $\Gamma$ has only a finite number of subgroups of given finite index, and also that the finite dimensional representations of $\Gamma$ form a sufficient family, i.e., separate points of $C^{*}(\Gamma)$.

To begin, we will show that all finite dimensional representations of $\Gamma$ are monomial.

Consider first the one dimensional characters of $\Gamma$. They form, of course, the dual group of $\Gamma / \Gamma^{(2)}$. But $\Gamma / \Gamma^{(2)} \cong\left(\Gamma / \Gamma_{s}^{(2)}\right) \times\left(\Gamma_{s}^{(2)} / \Gamma^{(2)}\right)$, the first factor being a lattice, the second a finite group. Any character in $\left(\widehat{\Gamma / \Gamma_{s}^{(2)}}\right)$ is the restriction of a character of $\mathscr{N}$, and so may be extended to a character of any $\Gamma^{\prime}$ containing $\Gamma$.

Proposition 1. A finite dimensional irreducible representation $U$ of $\Gamma$ may be written $U=\chi \otimes V$, where $\chi \in\left(\widehat{\Gamma / \Gamma_{s}^{(2)}}\right)$, and $V$ is a representation of a finite quotient group.

Proof. We induce on $\operatorname{rank}(\Gamma)(=\operatorname{dim} \mathscr{N})$. Choose $x \in \mathscr{Z}^{(2)}(\Gamma)-$ $\mathscr{Z}(\Gamma)$, and choose $y \in \Gamma$ such that $(x, y)=z \neq e$. Now $U_{\mid \mathscr{x}(\Gamma)}$ defines a character $\psi$ on $\mathscr{Z}(\Gamma)$, and $U\left(x y x^{-1}\right)=\psi(z) U(y)$. Hence, the spectrum of $U(y)$, which is a finite set, is invariant when multiplied by $\psi(z)$. Hence $\psi\left(z^{n}\right)=1$ for some $n$. Let $Z$ be the saturated subgroup generated by $z$, and let $\Gamma^{\prime} \subseteq \Gamma$ be a normal subgroup of finite index, such that $\psi_{\mid Z \cap \Gamma^{\prime}}$ is trivial. Then if $U^{\prime}$ is an irreducible component of $U_{\mid \Gamma^{\prime}}, U^{\prime}$ may be considered as a representation of $\Gamma^{\prime} / Z \cap \Gamma^{\prime}$, and so may be written $U^{\prime}=\chi^{\prime} \otimes V^{\prime}$. Extend $\chi^{\prime}$ to a character $\chi$ of $\Gamma / \Gamma_{s}^{(2)}$. Consider $V=\bar{\chi} \otimes U$ (-denotes the conjugate, or inverse, character). Then $V^{\prime}$ is a subrepresentation of $V_{\mid \Gamma^{\prime}}$. If $H$ is a normal subgroup of finite index in $\Gamma$, such that $H \cap \Gamma^{\prime} \cong \operatorname{ker} V^{\prime}$, then $V$ is seen to be trivial on $H \cap \Gamma$. Writing $U=\chi \otimes V$ gives the result.

CoRollary. All finite dimensional irreducible representations $U$ of $\Gamma$ are induced by one dimensional representations of subgroups of $\Gamma$.

Proof. Write $U=\chi \otimes V$. By the classical result for finite groups, $V=V^{\psi, \Gamma^{\prime}}, \psi$ being a one dimensional character of $\Gamma^{\prime} \leqq \Gamma$. Putting $\chi^{\prime}=\chi_{\mid \Gamma^{\prime}}$, the character formula for induced representations shows $U=U^{\chi^{\prime} \psi, \Gamma^{\prime}}$. 
Now we take a more global point of view towards the representations of $\Gamma$. For this, we assume $\Gamma$ is e.e., and write $L=\log \Gamma$.

If $L^{\prime} \leqq L$ is a sublattice, we denote by $r_{L^{\prime}}$ or $r$, when no confusion will arise, for the restriction map from $\hat{L}$ to $\hat{L}^{\prime}$. We say $\psi \in \hat{L}$ is rational on $L^{\prime}$ if $r(\psi)$ is a torsion element. Clearly $\psi$ is rational on $L^{\prime}$ if and only if it is rational on $L_{s}^{\prime}$, if and only if $\psi$ is identically one on $n L^{\prime}$ for some $n$. If $L^{\prime}$ is e.e., and $\psi$ is rational on $L^{\prime}$, we will also say $\psi$ is rational on $\Gamma^{\prime}=\exp L^{\prime}$.

LEMma 1. The orbit of $\psi \in \hat{L}$ under $\operatorname{Ad}^{*} \Gamma$ is finite if and only if $\psi$ is rational on $\log \Gamma_{s}^{(2)}$.

Proof. By C. H., given $y \in L, \delta \in \Gamma, y-\operatorname{Ad} \delta^{n}(y) \in n \log \Gamma_{s}^{(2)}$. Therefore, if $\psi$ is trivial on $n \log \Gamma_{s}^{(2)}, \psi$ is invariant by $\operatorname{Ad}^{*} \exp (n L)$, so $\#\left(\operatorname{Ad}^{*} \Gamma(\psi)\right) \leqq \#(L / n L)$, which is finite. On the other hand, there is a largest $\operatorname{Ad} \Gamma$ invariant sublattice $L^{\prime}$ on which $\psi$ is rational. $\Gamma^{\prime}=\exp L^{\prime}$ is then a saturated normal subgroup of $\Gamma$. If $\Gamma^{\prime} \nsupseteq \Gamma_{s}^{(2)}$, then $\Gamma / \Gamma^{\prime}$ is nonabelian.

Consider $\pi: \Gamma \rightarrow \Gamma / \Gamma^{\prime}$, and choose $\delta \in \Gamma$, so that $\pi(\delta) \in \mathscr{Z}^{(2)}\left(\Gamma / \Gamma^{\prime}\right)-$ $\mathscr{z}\left(\Gamma / \Gamma^{\prime}\right)$. Then, by C. H., we see that for $y \in \log \Gamma, \operatorname{Ad} \delta^{n}(y)=$ $y+n[\log \delta, y]\left(\bmod L^{\prime}\right) . \quad$ By choice of $L^{\prime}$ and $\delta, \psi[\log \delta, y]$ is irrational for some $y$. For this $y, \operatorname{Ad}^{*} \delta^{n}(\psi)(y)$ takes on an infinite number of values. A fortiori, $\operatorname{Ad}^{*} \Gamma(\psi)$ is infinite.

LEMma 2. Given $\psi \in \hat{L}$, the isotropy subgroup $\Gamma_{\psi}$ of $\psi$ under $\operatorname{Ad}^{*} \Gamma$ is an e.e. subgroup of $\Gamma$ and $\log \Gamma_{\psi}$ consists of all $y \in L$ such that $\psi([y, L]) \equiv 1$.

Proof. If $\delta \in \Gamma_{\psi}$, then $\operatorname{Ad}^{*} \delta \psi=\psi$, or $\left(\operatorname{Ad}^{*} \delta-I\right) \psi \equiv 1$. C. H. then shows, if $\Gamma$ is e.e., this is equivalent to $\psi([\log \delta, L]) \equiv 1$. If $a, b \in \log \Gamma_{\psi}$, then $[b, L] \subseteq k ! L$. Hence $\psi(1 / k ![b,[a, L]]) \equiv 1$. Interchanging $a$ and $b$, and using the Jacobi identity shows $\log \Gamma_{\psi}$ is e.e.

Lemma 3. If $\psi \in \hat{L}$ is $\mathrm{Ad}^{*} \Gamma$ invariant, then $\psi^{2}$ defines a onedimensional character on $\Gamma$.

Proof. C. H.

REMARK. This slight discrepancy between the $\psi$ 's which are class functions and those which are characters is the main source of the complexity of the statement of Theorem 1 .

To parallel Kirillov's theory, we need also a notion like "maximal 
subordinate subalgebra." This we now provide. Let $\mathscr{A}$ be a finite abelian group, and $B$ a biadditive, antisymmetric form on $\mathscr{A} \times \mathscr{A}$ into the circle. Then $B$ induces a homomorphism $\mathscr{A} \rightarrow \hat{\mathscr{A}}$ in the obvious way. The kernel $\mathscr{R}$ of this map is called the radical of $B$, and $B$ factors to a nondegenerate form $\widetilde{B}$ on $\mathscr{A} / \mathscr{R}=\mathscr{A}$. $\widetilde{B}$ can be polarized, that is, there are subgroups $\tilde{\mathscr{A}_{1}}, \tilde{\mathscr{A}_{2}}$ of $\tilde{\mathscr{A}}$ such that $\tilde{\mathscr{A}}=\tilde{\mathscr{A}_{1}} \times \tilde{\mathscr{A}_{2}}$, and $B$ is trivial on $\tilde{\mathscr{A}_{i}}$, so $B$ defines an isomorphism of $\tilde{\mathscr{A}_{1}}$ with $\hat{\mathscr{Z}_{2}}$, and vice-versa. A subgroup $\mathscr{A}_{1}$ of $\mathscr{A}$, containing $\mathscr{R}$, such that there $(\mathscr{A} / \mathscr{R})=\left(\mathscr{A} / \mathscr{A}_{1}\right)$ and $B / \mathscr{A}_{1}$ is trivial will be said to form half a polarization of $B$.

Now if $\tilde{\mathscr{A}}$ is a finite lie algebra, and $\psi \in \hat{\mathscr{A}}$, then $B_{\psi}\left(l_{1}, l_{2}\right)=$ $\psi\left(\left[l_{1}, l_{2}\right]\right)$ is a biadditive antisymmetric form, and $\mathscr{R}_{\psi}$, its radical, is a subalgebra of $\mathscr{A}$. It would be nice to find a subalgebra $\mathscr{P}$ such that $\mathscr{P} \supseteqq \mathscr{R}_{\psi}$, and $\mathscr{P} / \mathscr{R}_{\psi}$ formed half a polarization of $\hat{B}_{\psi}$ on $\mathscr{A} \mid \mathscr{R}_{\psi}$. If $\mathscr{P}$ exists, we will call it a polarizing subalgebra for $\psi$.

Lemma 4. If $\mathscr{A}$ is a nilpotent finite lie algebra, and $\psi \in \hat{\mathscr{A}}$, then polarizing subalgebras for $\psi$ exist.

REMARKS. The parallel result for real nilpotent lie algebras was essentially established by Kirillov [9] and emphasized by Brezin [4].

(b) This is a key place where we imitate Kirillov's inductive arguments.

Proof. We first assume a sublemma.

Sublemma. If $B$ is an antisymmetric biadditive form on the finite abelian group $\mathscr{A}$ into the circle, with radical $\mathscr{R}$, and $\pi: \mathscr{A} \rightarrow$ $\mathscr{A} \mid \mathscr{R}$ is the natural map, and if $\pi\left(\mathscr{A}_{1}\right), \pi(\mathscr{A})$ form a polarization of $\widetilde{B}$, for subgroups $\mathscr{A}_{i}$ containing $\mathscr{R}$, then $\sharp\left(\mathscr{A}_{1}\right)=\#\left(\mathscr{A}_{2}\right)$, and $\#(\mathscr{A} / \mathscr{R})=\#\left(\mathscr{A} / \mathscr{A}_{1}\right)$. Conversely, if $\#\left(\mathscr{A}_{1}\right)^{2}=\#(\mathscr{A}) \#(\mathscr{R})$, and $B$ is trivial on $\mathscr{A}_{1}$, then $\pi\left(\mathscr{A}_{1}\right)$ forms half of a polarization of $\widetilde{B}$.

Now consider $B_{\psi}$ on $\mathscr{A}$. Clearly $\mathscr{Z}(L)$, the center of $\mathscr{A}$ considered as lie algebra, is contained in $\mathscr{R} \psi$. Let $C=\operatorname{ker} \psi \cap \mathscr{\varkappa}(\mathscr{A})$. If $C \neq\{e\}$, then factor $\psi$ to $\psi^{\prime} \in \widehat{\mathscr{A} / \mathscr{C}}$. Then there is a subalgebra $\mathscr{P ^ { \prime }}$ of $\mathscr{A} / \mathscr{C}$, polarizing $\psi^{\prime}$ by induction. Then $\mathscr{P}$, the inverse image of $\mathscr{P}^{\prime}$ in $\mathscr{A}$, is seen by the sublemma to be a polarizing subalgebra for $\psi$.

If $\psi$ is faithful on $\mathscr{Z}(\mathscr{A})$, then $\mathscr{Z}$ must be cyclic. Let $z$ generate $\mathscr{Z}$. Pick $y \in \mathscr{Z}^{(2)}(\mathscr{A})-\mathscr{Z}(\mathscr{A})$, such that $p y \in \mathscr{Z}(\mathscr{A})$ for some prime $p$. Then $C(y)$, the centralizer of $y$ in $\mathscr{A}$, is an ideal of $\mathscr{A}$, of index $p$. For $C(y) \neq \mathscr{A}$, since $y \notin \mathscr{Z}(\mathscr{A})$, but $[y, \mathscr{A}] \subseteq$ $\mathscr{Z}(\mathscr{A})$, so the Jacobi identity implies $[\mathscr{A}, \mathscr{A}] \subseteq C(y)$, so $C(y)$ is an 
ideal. Pick $x \in \mathscr{A}-C(y)$. Then $[y, x]=m z$, for some $m \in Z$. Hence $[a y, b x]=a b m z$. In particular $[p y, x]=p m z=e$, so $p m$ is a multiple of the period of $z$, and $[y, p x]=p m z=e$. Therefore $p x \in C(y)$, and $x$ generates over $C(y)$, so $C(y)$ is as stated.

Let $\psi^{\prime}=\psi_{(C(y)}$. By induction $\psi^{\prime}$ admits a polarizing subalgebra $\mathscr{P}$. Clearly $y \in \mathscr{R}_{\psi}$, but $y \notin \mathscr{R}_{\psi}$, since $\psi([x, y]) \neq 1$ by construction. Hence, as $\sharp(\mathscr{P})^{2}=\#\left(\mathscr{R}_{\psi}, \#(C(y))\right.$, and $\#\left(\mathscr{R}_{\psi} / \mathscr{R}_{\psi}\right) \geqq p$, the sublemma shows $\mathscr{P}$ must polarize $\psi$.

These notions are easily transferable to $L=\log \Gamma$ for $\Gamma$ e.e. If $\psi \in \hat{L}$, then we may form $B_{\psi}$ just as in the finite case. By Lemma $2, \mathscr{R}_{\psi}=\log \Gamma_{\psi}$. Hence if $\psi$ is rational on $\Gamma^{(2)}, \mathscr{R}_{\psi}$ is of finite index in $L$. Moreover, by changing $\psi$ by a character of $\Gamma / \Gamma_{s}^{(2)}$, which does not alter $B_{\psi}$, we may assume $\psi$ is itself rational, and so by passing to a quotient algebra, may consider ourselves to be in the finite situation. In particular we may talk about polarizing subalgebras $P$ for $\psi$. The crucial relation that $P$ satisfies is here expressible by the formula $\sharp\left(P / \mathscr{R}_{\psi}\right)=\#(L / P)$. We have the following corollary to Lemma 4.

Corollary. Taking $L=\log \Gamma$, if $\psi \in L$ is rational on $\Gamma^{(2)}$, then there are e.e. polarizing subalgebras $P$ for $\psi$.

Proof. As indicated above, we may assume $\psi$ is rational on $L$. Put $L^{\prime}=(1 / k !) L$. Let $\psi^{\prime}$ be the element of $L^{\prime}$ defined by $\psi^{\prime}(y)=$ $\psi\left(k !^{2} y\right)$ for $y \in L^{\prime}$. Passing to a finite quotient, applying Lemma 4, and lifting back, there is $P^{\prime} \subseteq L^{\prime}$, a polarizing subalgebra for $\psi^{\prime}$. But then $P=k ! P^{\prime}$ is an e.e. polarizing subalgebra for $\psi$.

LEMMA 5. If $P$ is an e.e. polarizing subalgebra for $\psi \in \hat{L}, \psi$ rational on $\log L_{s}^{(2)}$, and if $\pi=\exp P$, then $\operatorname{Ad}^{*} \pi(\psi)$ consists of all $\phi \in L$ such that $\phi_{\mid P}=\psi_{\mid P}$.

Proof. Since $P$ is e.e., and polarizes $\psi$, C. H. shows $\operatorname{Ad}^{*} x(\psi)_{\mid P}=$ $\psi_{\mid P}$ for any $x \in \pi$. On the other hand, the remark just above shows by counting that $\mathrm{Ad}^{*} \pi(\psi)$ consists of all possible $\varphi$.

Now we need a lemma on induced representations. We say representations $U, V$, are disjoint if they have no common subrepresentation.

LEMma 6. If $G$ is a finite group, $H_{1}, H_{2}$ subgroups, $V_{i}$ representations of $H_{i}$ with characters $\psi_{i}$, then $U_{1}=U^{v_{1}, H_{1}}$ and $U_{2}=$ $U^{V_{2} H_{2}}$ are disjoint if and only if $\mathrm{Ad}^{*} x\left(\dot{\psi}_{1}\right)$ and $\mathrm{Ad}^{*} y\left(\dot{\psi}_{2}\right)$ are orthogonal (in $l_{2}(G)$ ) for all $x, y$ in $G$. $U_{1}$ is irreducible if and only if $\mathrm{Ad}^{*} x\left(\dot{\psi}_{1}\right)$ is orthogonal to $\dot{\psi}_{1}$ for all $x \in G-H_{1}$. 
Proof. Let $\varphi_{i}$ be the character of $U_{i}$. By the formula for induced characters, $\varphi_{i}^{-}$is a sum of terms $\mathrm{Ad}^{*} x\left(\dot{\psi}_{i}\right)$. As $U_{1}$ and $U_{2}$ are disjoint if and only if $\varphi_{1}$ and $\varphi_{2}$ are orthogonal, and since the inner product of $\mathrm{Ad}^{*} x\left(\dot{\psi}_{1}\right)$ and $\mathrm{Ad}^{*} y\left(\dot{\psi}_{2}\right)$ is nonnegative, since they are positive definite functions, the first statement is clear. By a theorem of Mackey [10], $U$ is irreducible if and only if the representation $V_{1}^{x}$ of $x^{-1} H_{1} x=H_{1}^{1}$, given by $h^{1} \rightarrow V\left(x h^{1} x^{-1}\right)$ when restricted to $H_{1}^{1} \cap H=F$, is disjoint from $V_{1}$ restricted to $F$, for any $x \in G-$ $H_{1}$. This happens exactly when $\dot{\psi}_{1 \mid F}$ and $\mathrm{Ad}^{*} x\left(\dot{\psi}_{1}\right)_{\mid F}$ are orthogonal in $l_{2}(F)$. But off $F$, either $\dot{\psi}_{1}$ or $\operatorname{Ad}^{*} x\left(\dot{\psi}_{1}\right)$ vanishes. Hence orthogonality on $F$ implies orthogonality on $G$, and conversely. The result follows.

We now classify the finite dimensional representations of $\Gamma$.

THEOREM 1. Let $\Gamma$ be an e.e., discrete, finitely generated, torsion free-nilpotent group. Let $L=\log \Gamma, L^{\prime}=2 L, \Gamma^{\prime}=\exp L^{\prime}$, $L_{s}^{(2)}=\log \Gamma_{s}^{(2)}$, and $n \Gamma=\exp (n L)$ for $n>2$.

(a) Let 0 be a finite $\mathrm{Ad}^{*} \Gamma$ orbit in $\hat{L}$, and $\psi \in 0$. Let $n$ be the period of $\psi_{i L_{s}}^{(2)}$. Call $n$ the period of 0 . If $n$ is odd, then $a$ finite dimensional irreducible representation $U_{0}$ of $\Gamma$ may be associated to 0 in the following manner. If $P$ is an e.e. polarizing subalgebra for $\psi$, and $\pi=\exp P$, put $\psi_{\mid \pi}=\widetilde{\psi}$. Then $U_{0}=U \widetilde{\psi}$. The dimension of $U_{0}$ is $\#(0)^{1 / 2}$, and the character $\xi_{0}$ of $U_{0}$ is given by $\xi_{0}=\#(0)^{-1 / 2} \sum_{\varphi \in 0} \varphi$. All representations of the form $\chi \otimes V$, with $\chi \in$ $\widehat{\Gamma / \Gamma_{s}^{(2)}}$, and $V$ defined modulo $n \Gamma, n$ odd, are realized in this manner.

(b) In general, there is a surjective map from finite dimensional irreducible representations of $\Gamma^{\prime}$ to finite $\mathrm{Ad}^{*} \Gamma$ orbits in $\hat{L}^{\prime}$. This map is at most $\#\left(\Gamma / \Gamma^{\prime}\right)$-to-one. If $\left\{U_{i}\right\}_{i=1}^{l}$ map onto the orbit 0 , then the $U_{i}$ are permuted transitively among themselves by the action $\mathrm{Ad}^{*} \Gamma / \Gamma^{\prime}$, so that they define a point in $M\left(\Gamma^{\prime}, \Gamma\right) . \quad l$ is a power of 2 , and if $m$ is the common dimension of the $U_{i}, l m^{2}=\#(0)$. The sum of the characters $\xi_{i}$ of the $U_{i}$ is given by the formula $\Sigma \xi_{i}=m^{-1} \sum_{\varphi \in 0} \varphi$.

REMARK. For two-step nilpotent groups, it is not hard to see that the correspondence is even more immediate: the functions $\phi$ in an orbit 0 span the annihilator (in $l_{\infty}(\Gamma)$ ) of the kernel of $U_{0}$. This appears not to be true in general. The precise role played by an individual $\psi \in \hat{L}$ is as yet unclear.

Proof. It may be verified using C. H., that for $\delta \in \Gamma, \log (\delta n \Gamma)=$ $\log \delta+\log n \Gamma$. Therefore, if $A_{n}$ is the group of elements of $\hat{L}$ of order dividing $n, A_{n}$ can be naturally interpreted as a set of func- 
tions on $\Gamma / n \Gamma$, and in fact, they form an orthogonal basis for $l_{2}(\Gamma / n \Gamma)$. More generally, if $\chi \in \widehat{\Gamma / \Gamma_{s}^{(2)}}, \chi A_{n}$ may be considered as an orthogonal basis for the induced module $U^{x_{n}}$, where $\chi_{n}=\chi_{i n_{n} \Gamma}$. Moreover, given 0 with odd period $n$, it is clearly a subset of $\chi A_{n}$ for suitable $\chi$.

Now take 0 with odd period, and $\psi \in 0$, and $P$, an e.e., polarizing subalgebra for $\psi$, and put $\pi=\exp P$. By Lemma $3, \psi^{2}$ is a character on $\pi$, and since $\psi_{\mid L^{(2)}}$ has odd period, it follows that $\psi$ itself defines a character $\psi_{0}$ on $\pi$. Consider $U_{0}=U^{\psi_{0}, \pi}$. This is a representation of $\Gamma$ with dimension $\#(\Gamma / \pi)=\#(0)^{1 / 2}$. It follows from Lemma 5 , and the orthogonality properties of characters on finite abelian groups, that $\#(0)^{-1 / 2} \sum_{\varphi \in \mathrm{Ad}^{*} \pi(\psi)} \varphi=\dot{\psi}_{0}$. From this, we see if $\delta \in \Gamma-\pi, \operatorname{Ad}^{*} \delta\left(\dot{\psi}_{0}\right)$ is then a similar sum over a subset of 0 , disjoint from $\operatorname{Ad}^{*} \pi(\psi)$, and hence is orthogonal to $\dot{\psi}_{0}$. Lemma 6 now shows $U_{0}$ is irreducible. The general formula for induced characters is seen to reduce to the stated one in this case. The formula then guarantees that $U_{0}$ is independent of $\psi$ and $\pi$, and depends only on 0 . Finally, since $\operatorname{dim} U_{0}^{2}=\#(0)$, the total dimension of representations coming from orbits in $A_{n}$ is $\#\left(A_{n}\right)=\#(\Gamma / n \Gamma)$. This shows that all representations of the stated form arise in this manner. This establishes (a).

For (b), we refine these considerations. Take $\Gamma, \Gamma^{\prime}$, and a finite $\operatorname{Ad}^{*} \Gamma$ orbit 0 in $\hat{L}^{\prime}$, and $\psi \in 0$. Since $\left[L^{\prime}, L^{\prime}\right] \subseteq 2 k ! L^{\prime}$, the proof of the corollary to Lemma 4 shows we can find $P^{\prime} \subseteq L^{\prime}$, which polarizes $\psi$, and such that $\left[P^{\prime}, P^{\prime}\right] \subseteq 2 k ! P^{\prime}$. Put $P=(1 / 2) P^{\prime}$. Then $P$ is e.e., and $P^{\prime}$ is an ideal in $P$. Write $\pi^{\prime}=\exp P^{\prime}$, and $\pi=\exp P$. As before $\operatorname{Ad}^{*} \pi^{\prime}(\psi)=0_{\pi^{\prime}}$ consists of all $\varphi \in L^{\prime}$ such that $\varphi_{\mid P^{\prime}}=\psi_{\mid P^{\prime}}$. However $\psi_{\left(\pi^{\prime}\right.}$ may not define a character on $\pi^{\prime}$. We account for this discrepancy in this way. Consider the form $B_{\psi}^{\prime}(x, y)=\psi(1 / 2[x, y])$ on $P^{\prime}$. If $B_{\psi}^{\prime}$ is trivial, then $\psi$ does define a character on $\pi^{\prime}$, and we may proceed as before. If $B_{\psi}^{\prime}$ is not trivial, let $A^{\prime} \subseteq P^{\prime}$ denote its radical. Then $A^{\prime}$ is an e.e. ideal in $P^{\prime}$, and $A^{\prime} \supseteqq 2 P^{\prime} \supseteq\left[P^{\prime}, P^{\prime}\right]$. Let $A=(1 / 2) A^{\prime}, \mathscr{A}=\exp A, \mathscr{A}^{\prime}=\exp A^{\prime}$. Also, let $\Gamma^{\prime \prime}$ be the subgroup of $\Gamma$ generated by $\Gamma^{\prime}$ and $\pi$, and let $H=\pi \cap \Gamma^{\prime}$. We verify the following facts:

(1) $\psi_{\mathscr{M}}$ defines a character $\psi_{0}$ of $\mathscr{A}^{\prime}$.

(2) $O_{\pi}=\operatorname{Ad}^{*} \pi(\psi)$ consists of all $\varphi \in \hat{L}^{\prime}$ such that $\varphi_{\mid \mathscr{A}^{\prime}}=\psi_{0}$.

(3) $\mathscr{A}$ is the subgroup of $\pi$ consisting of elements $a$ such that $\operatorname{Ad}^{*} a(\psi)_{\mid P}=\psi_{\mid P}$.

(4) $\dot{\psi}_{0}=\#\left(\Gamma^{\prime} / \mathscr{A}^{\prime}\right)^{-1} \sum_{\varphi \in 0_{\pi}} \varphi$.

(5) $\sharp\left(\Gamma / \Gamma^{\prime \prime}\right)=\#\left(H / \pi^{\prime}\right)$ and $\sharp\left(\Gamma^{\prime \prime} / \Gamma^{\prime}\right)=\#(\pi / H)$.

(6) $\pi^{\prime} \subseteq H$. If $h \in H-\pi^{\prime}$, then $h \notin \mathscr{A}$.

(1) is clear by the definition of $\mathscr{f}^{\prime}$ and Lemma 3 . Since $\#(\pi / \mathscr{A})=\#\left(\pi^{\prime} / \mathscr{A}^{\prime}\right),(2)$ is implied by the parallel fact for $0_{\pi^{\prime}}$, together 
with (3). (3) follows from the definition of $A$, using C. H. (4) is immediate from (2) and the orthogonality properties of the $\phi$ 's. (5) is clear from the definitions, as is the first statement of (6). The second statement in (6) follows because $P^{\prime}$ polarizes $\psi$, and so constitutes a maximal subalgebra of $L^{\prime}$ on which $B_{\psi}$ is trivial. We now consider the induced representation $V=V^{\psi_{0}, \mathscr{Q}^{\prime}}$ of $\pi^{\prime}$. Since $P^{\prime}$

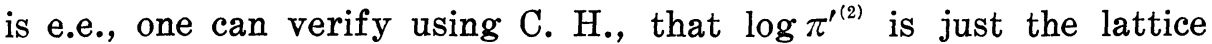
generated by $\left[P^{\prime}, P^{\prime}\right]$. Since $\psi$ must be trivial on this lattice, it follows that $V$ is just a sum of $\#\left(\pi^{\prime} / \mathscr{A}^{\prime}\right)$ one dimensional representations with characters $\left\{\chi_{i}\right\}$. Consider the induced representation $U_{i}=U^{\chi_{i}, \pi^{\prime}}$ of $\Gamma^{\prime}$. $\quad U_{i}$ is irreducible. Because, if $\delta \in \Gamma^{\prime}-H$, then (4) above shows $\operatorname{Ad}^{*} \delta\left(\dot{\psi}_{0}\right)$ is orthogonal to $\dot{\psi}_{0}$, so a fortiori, $\operatorname{Ad}^{*} \delta\left(\dot{\chi}_{i}\right)$ is orthogonal to $\dot{\chi}_{i}$. On the other hand, if $\delta=h \in H-\pi^{\prime}$, then $\operatorname{Ad}^{*} h\left(\chi_{i}\right)(p)=$ $\chi_{i}(p) \psi_{0}((h, p))$, for $p \in \pi^{\prime}$. Hence $\operatorname{Ad}^{*} h\left(\chi_{i}\right)=\chi_{i}$ means $\psi_{0}((h, p))=\psi_{0}$ $([\log h, \log p])=1$ for all $p$ in $\pi^{\prime}$, which is not true since $h \notin \mathscr{A}$, by (6) above.

Therefore, by the transitivity properties of induced representations, the representation $U=U^{\psi_{0}, \varkappa^{\prime}}$ of $\Gamma^{\prime}$, decomposes into a direct sum of $\#\left(\pi^{\prime} / \mathscr{L}^{\prime}\right)$ factors $U_{i}$, each of dimension $\#\left(\Gamma^{\prime} / \pi^{\prime}\right)$. However, the $U_{i}$ are not all distinct. From the discussion just above, we see $U_{i}$ and $U_{j}$ are equivalent if and only if $\chi_{i}$ and $\chi_{j}$ are in the same $\mathrm{Ad}^{*} H / \pi^{\prime}$ orbit. But we also saw above that $\mathrm{Ad}^{*} H / \pi^{\prime}$ acts freely and faithfully on the $\left\{\chi_{i}\right\}$. Hence each equivalence class of representations occurs $\#\left(H / \pi^{\prime}\right)$ times, and the number $l$ of equivalence classes is $l=\#\left(\pi^{\prime} / \mathscr{A}^{\prime}\right) \#\left(H / \pi^{\prime}\right)^{-1}$. Both these numbers are powers of two, and $l$ is clearly $\leqq \#\left(\Gamma / \Gamma^{\prime}\right)$. Now $\mathrm{Ad}^{*} \pi / \pi^{\prime}$ permutes transitively the $\left\{\chi_{i}\right\}$, because $\pi / \mathscr{A}$ acts freely on the $\left\{\chi_{i}\right\}$, for the same reason that $H / \pi^{\prime}$ does, and $\#(\pi / \mathscr{A})=\#\left(\pi^{\prime} / \mathscr{A}^{\prime}\right)$. Therefore $\mathrm{Ad}^{*} \Gamma^{\prime \prime} / \Gamma^{\prime}$ permutes transitively the classes of the $\left\{U_{i}\right\}$; so they define a point in $M\left(\Gamma^{\prime}, \Gamma^{\prime \prime}\right)$.

We now compute the character $\xi$, of $U$. If $\left\{h_{i}\right\}$ is a set of coset representatives for $\mathscr{A}^{\prime}$ in $H$, and $\left\{\delta_{j}\right\}$ is a set of coset representatives for $H$ in $\Gamma^{\prime}$, then $\left\{\delta_{j} h_{i}\right\}$ are a set of coset representatives of $\mathscr{A}^{\prime}$ in $\Gamma^{\prime}$, and $\xi=\sum_{i, j} \mathrm{Ad}^{*} \delta_{j} h_{i}\left(\dot{\psi}_{0}\right)$

$$
\begin{aligned}
& =\sum_{i, j} \operatorname{Ad}^{*} \delta_{j} h_{i}\left(\#\left(\Gamma^{\prime} / \mathscr{C}^{\prime}\right)^{-1} \sum_{\varphi \in 0_{\pi}} \varphi\right) \\
& =\sum_{j} \operatorname{Ad}^{*} \delta_{j}\left(\left(\#\left(H / \mathscr{C}^{\prime}\right) \#\left(\Gamma^{\prime} / \mathscr{\mathscr { C } ^ { \prime }}\right)^{-1}\right) \sum_{\varphi \in 0_{\pi}} \varphi\right) \\
& =\#\left(\Gamma^{\prime} / H\right)^{-1} \sum_{\varphi \in 0^{\prime \prime}} \varphi, \text { where } 0^{\prime \prime}=\operatorname{Ad}^{*} \Gamma^{\prime \prime}(\psi),
\end{aligned}
$$

since $\left\{\delta_{j}\right\}$ also form a set of coset representatives for $\pi$ in $\Gamma^{\prime \prime}$. Since each representation involved occurs $\#\left(H / \pi^{\prime}\right)$ times, $\xi_{0}$, the character of the quasiequivalent multiplicity free representation is 


$$
\xi_{0}=\#\left(\Gamma^{\prime} / H\right)^{-1} \#\left(H / \pi^{\prime}\right)^{-1} \sum_{\varphi \in 0^{\prime \prime}} \varphi=\#\left(\Gamma^{\prime} / \pi^{\prime}\right)^{-1} \sum_{\varphi \in 0^{\prime \prime}} \varphi=\left(\operatorname{dim} U_{i}\right)^{-1} \sum_{\varphi \in 0^{\prime \prime}} \varphi \text {. }
$$

Let $S, S^{\prime}, S^{\prime \prime}$ denote the isotropy subgroups of $\psi$ under $\operatorname{Ad}^{*} \Gamma$, $\mathrm{Ad}^{*} \Gamma^{\prime \prime}$, and $\mathrm{Ad}^{*} \Gamma^{\prime}$. We have $\sharp\left(\Gamma^{\prime} / S^{\prime}\right)=\sharp\left(\Gamma^{\prime} / \pi^{\prime}\right)^{2}$ since $P$ polarizes $\psi$. Moreover, since $\mathrm{Ad}^{*} \mathscr{A}$ fixes $\psi_{\mid p^{\prime}}$, we have $\#\left(S^{\prime \prime} / S^{\prime}\right)=\#\left(\mathscr{A} / \pi^{\prime}\right)$. From these relations, we see $\#\left(0^{\prime \prime}\right)=\#\left(\Gamma^{\prime \prime} / S^{\prime \prime}\right)=\#\left(\Gamma^{\prime} / S^{\prime}\right) \#\left(\Gamma^{\prime \prime} / \Gamma^{\prime}\right) \#\left(\mathscr{A} / \pi^{\prime}\right)^{-1}$. But $\sharp\left(\Gamma / \Gamma^{\prime \prime}\right) \#\left(\Gamma^{\prime \prime} / \Gamma^{\prime}\right)=\#\left(\Gamma / \Gamma^{\prime}\right)$ and $\#(\pi / \mathscr{A}) \#\left(\mathscr{C} / \pi^{\prime}\right)=\#\left(\pi / \pi^{\prime}\right)$. But as the two right hand sides are equal, and as $\#(\pi / \mathscr{A})=\#\left(\pi^{\prime} / \mathscr{A}^{\prime}\right)$, and using (5), we have $\#\left(0^{\prime \prime}\right)=l\left(\operatorname{dim} U_{i}\right)^{2}$.

Thus the set $0^{\prime \prime}$ may be associated with representations $\left\{U_{i}\right\}$ satisfying all the claims of the theorem. But 0 is just a union of $0_{j}^{\prime \prime}=\mathrm{Ad}^{*} \delta\left(0^{\prime \prime}\right)$, for $\delta \in \Gamma$, and the representations associated to different $0_{j}^{\prime \prime}$ are clearly disjoint, again by orthogonality. Hence the theorem holds for 0 , and a counting argument again shows all finite dimensional representations are obtained thus.

We remark that the character formula allows effective computation of the irreducible decomposition of induced representations and tensor products of representations of $\Gamma$. For example, let $F \subseteq \Gamma$ be e.e., and contain $n \Gamma$ for $n$ odd, and let $\psi$ be a one dimensional character of $F / n \Gamma$. Then $U^{\psi, F}$ contains $U_{0}$ to multiplicity $\#(0)^{-1 / 2}$ $\#\left(0 \cap r^{-1}(\psi)\right)$, where $r=r_{\log F}$.

III. Primitive ideal spaces. Let $G$ be a countable discrete group. Then $M(G)$ is compact. According to Fell [6] and Prosser [12], if $I$ is a closed two-sided *-ideal of $C^{*}(G)$, the map which sends $I$ to $I^{\perp}$, the $\mathrm{Ad}^{*} G$ invariant weak *-closed cone of positive definite functions in $l_{\infty}(G)$ which annihilate $I$ is a bijective correspondence, and the hull-kernel topology goes over to the weak *-topology, which in this case is equivalent to the topology of pointwise convergence. Call $I^{\perp}$ a dual ideal. We will use the correspondence $I \leftrightarrow I^{\perp}$ to consider $I^{\perp}$ as defining or being a closed set in $M(G)$. If $I^{\perp}$ is a minimal dual ideal, then $I$ is maximal, and the corresponding set in $M(G)$ is a closed point. Recall $M(G)$ is $T_{1}$ if and only if all primitive ideals are maximal. If $S$ is a dual ideal, and $S=I^{\perp}$, we will also write $I=S^{\perp}$.

Let $H \cong G$ be a normal subgroup. We have already introduced $\mathrm{Ad}^{*} G / H$ and $M(H ; G)$. If $S$ is a dual ideal of $G$, let $r_{H}(S)=r(S)$ be the dual ideal of $H$ consisting of restrictions to $H$ of functions in $S$. If $T$ is a dual ideal of $H$, let $i(T)$ be the dual ideal of $G$ generated by all functions $\dot{f}, f \in T$. The following relations are immediate:

(1) $r(i(T)) \supseteqq T$.

(2) $r(S)$ is $\mathrm{Ad}^{*} G / H$ invariant, and $T=r(i(T))$ if and only if $T$ is $\mathrm{Ad}^{*} G / H$ invariant. 
(3) $r \circ i \circ r=r$ and $i \circ r \circ i=i$.

Moreover, if $G$ is amenable (of if just $G / H$ is amenable) (as is the case for our $\Gamma$ ) then it follows from results of Greenleaf [8] that

(4) $i(r(S)) \supseteqq S$.

(5) $S=i(r(S))$ if and only if $f \in S$ implies $\left(\dot{f}_{\mid H}\right) \in S$. If $S=i(r(S))$, we will say, $S$ is induced from $H$, or $S$ may be reduced to $H$. We do not distinguish notationally between this operation $r$, and the $r$ previously defined for restrictions of characters to sublattices, because they are in fact the same operation.

$r$ and $i$ are closely related to familiar operations on representations.

Lemma 7. If $I$ is a closed ${ }^{*}$ ideal of $C^{*}(G)$, and $U$ is a representation of $G$ with kernel $I$, then $U_{\mid H}$ has kernel $J=r\left(I^{\perp}\right)^{\perp}$. If $J$ is a closed *ideal of $C^{*}(H), V$ a representation of $H$ with kernel $J$, then $U^{V, H}$ has as kernel in $C^{*}(G)$ the ideal $I=i\left(J^{\perp}\right)^{\perp}$.

Proof. This is straight forward from the definitions, plus the well known fact ([6]) that if $U$ is a representation of $G$, on the Hilbert space $\mathscr{H}$, with kernel $I \subseteq C^{*}(G)$, then $I^{\perp}$ is generated as dual ideal by the positive definite functions $g \rightarrow(U(g) x, x), x \in \mathscr{H}$.

Lemma 8. If $I \in M(G)$, then $r\left(I^{\perp}\right)$ defines a point in $M(H, G)$.

Proof. $M(H, G)$ is the quasiorbit space of the action $\mathrm{Ad}^{*} G / H$ on $M(H)$. Let $U$ be an irreducible representation of $G$ on $\mathscr{H}$, with kernel $I$, and consider $U_{\mid H}$. Let $\mathscr{A}$ be the von Neumann algebra generated by $U(H)$, and $C$ the center of $\mathscr{A}$. If $B \subseteq M(H)$ is a closed subset, let $I_{B}$ be the intersection of the ideals in $B$. Let $\mathscr{H}_{B}$ be the null space of $U\left(I_{B}\right)$. Then $P_{B}$, orthogonal projection onto $\mathscr{H}_{B}$, is in $C$. Since $I_{B_{1} \cup B_{2}}=I_{B_{1}} \cap I_{B_{2}}, \mathscr{C}_{B_{1} \cup B_{2}}=\mathscr{H}_{B_{1}}+\mathscr{C}_{B_{2}}$, so $P_{B_{1} \cup B_{2}}=\sup \left(P_{B_{1}}, P_{B_{2}}\right)$. Therefore, we may extend this to a map from the $\sigma$-Boolean algebra of Borel sets of $M(H)$ to the projections in $C$. (See [7].)

Take $x \in M(H)-r\left(I^{\perp}\right)$. Since $M(H)$ is compact, and $r\left(I^{\perp}\right)$ is closed, there exists a neighborhood $A$ of $x$, disjoint from $r\left(I^{\perp}\right)$. Therefore $P_{A}+1-P_{M-A}$ is zero, since $M-A \supseteqq r\left(I^{\perp}\right)$, and $P_{r\left(I^{\perp}\right)}=1$, by Lemma 7. On the other hand, take $x \in r\left(I^{\perp}\right)$, and $A$ any neighborhood of $x$. Then $P_{A}$ is not zero. For if it were, then $P_{M-A}=1$, so the null space of $U\left(I_{M-A}\right)$ would be all of $\mathscr{H}$, which would mean $I_{M-A} \subseteq \operatorname{ker} U_{\mid N}$ or $M-A \supseteqq r\left(I^{\perp}\right)$, contrary to the choice of $x$ and $A$.

Now choose $\left\{x_{i}\right\}_{i=1}^{\infty} \in \mathscr{H}$, which span, and such that $\Sigma\left\|x_{i}\right\|^{2}=1$. Define $M(B)=\Sigma\left(P_{B} x_{i}, x_{i}\right)$ for any borel set $B \subseteq M$. This defines a borel measure on $M$. Clearly $M(B)=0 \Leftrightarrow P_{B}=0$. We conclude that $\mu$ is concentrated on $r\left(I^{\perp}\right)$, and on no smaller closed set. Since 
$U$ is irreducible for $G, \mu$ is also seen to be ergodic under $A^{*} G / H$. $M(H)$ is known to be an analytic Borel space, and in particular, countably separated. Now apply Lemma 1.1 of Effros and Hahn [5] to conclude that $\mu$ is concentrated in a unique quasiorbit for $\mathrm{Ad}^{*} G / H$. From the above, $r\left(I^{\perp}\right)$ must be this quasiorbit.

Thus $r$ becomes a map $r: M(G) \rightarrow M(H, G)$, and $M(H, G)$ is seen to be the quotient of $M(G)$ by the equivalence relation defined by restriction of dual ideals of $G$ to $H$. This justifies the term, relative primitive ideal space. $r$ is clearly continuous.

Addendum. The author is grateful to the reference for raising the following two points bearing on the justification of the term "relative primitive ideal space." The points are:

(1) Is $r: M(G) \rightarrow M(H)$ surjective?

(2) Is $r$ an open map? That is, is the topology of $M(H, G)$ as the set of ideals of $C^{*}(H)$ primitive relative to the action of $\operatorname{Ad}^{*} G / H$ the same as the topology of $M(H, G)$ as the set of classes of primitive ideals of $C^{*}(G)$ relativized to $H$ ?

The answer to (1) is always yes. I do not know the answer to (2) in general, but if $G / H$ is amenable the answer is yes. Amenability is certainly not necessary, because one can take $H$ to be finite for example. However, amenability covers the case at hand.

The proof of surjectivity goes as follows. Let $\widetilde{0} \subseteq M(H)$ be an $\operatorname{Ad}^{*} G / H$ quasiorbit. Let $J \in M(H)$ generate $\widetilde{0}$ (i.e., $\operatorname{Ad}^{*} G / H(J)$ is dense in $\widetilde{0})$. Let $V$ be an irreducible representation of $H$ corresponding to $J$, on the Hilbert space $\mathscr{H}$. Consider the positive definite function $f(h)=(V(h) u, u)$, defined by the unit vector $u \in \mathscr{K}$. $f$ is an extreme point of the states of $C^{*}(H)$. Now $r(i(J))=\widetilde{0}$, and $i(J)$ is a dual ideal of $C^{*}(G)$; so the states in $i(J)$ form a support of the states of $C^{*}(G)$ (see [12]). Thus we may choose an extreme point $g$ of the states of $C^{*}(G)$, such that $g \in i(J)$ and $g_{\mid H}=f$. The dual ideal generated by $g$ will then define a point of $M(G)$ whose image under $r$ is $\tilde{0}$.

We pass to point (2). The inverse images under $r$ of the closed sets of $M(H, G)$ are the sets of the form $S_{J}=\{I \in M(G) ; r(I) \subseteq J\}$, $J \subseteq M(H), J$ closed and $\mathrm{Ad}^{*} G / H$ invariant. Now let $T$ be any closed $r$-saturated set in $M(G)$, and let $J=r(T)$. Then I claim $i(J) \subseteq T$ always. To see this, let $I$ be any point of $T$. Then to say that $T$ is $r$-saturated is to say that for any $I^{\prime}$, such that $r\left(I^{\prime}\right)=r(I), I^{\prime} \in T$. Now the proof of surjectivity given above may be refined by looking at all possible functions $g$ chosen as in that argument. If $f$ is an extreme point of the states in $r(I)$, the Krein-Milman theorem implies $\dot{f} \in r^{-1}(r(I))$, hence $f \in T$. Applying the Krein-Milman theorem again shows $\dot{g} \in T$ for any $g \in r(I)$, and apply- 
ing it again shows $i(r(T)) \cong T$ as claimed. Thus always $S_{J} \supseteqq T \supseteqq$ $i(J)$. But now if $G / H$ is amenable, $i(r(T)) \supseteqq T$. Hence $T=i(r(T))=$ $S_{J}$ and the topologies agree in this case.

We also note that it is only the nonmaximal primitive ideals which cause problems in these considerations. If one is interested in maximal ideals only, then Lemma 8 and the above discussion become trivial. $r$ exists and is open automatically.

IV. Structure of ideals of $C^{*}(\Gamma)$.

Lemma 9. (a) Let $\Gamma_{1} \subseteq \Gamma$ be a subgroup, and $n\left(\Gamma_{1}\right)$ its normalizer in $\Gamma$. Then $n\left(\Gamma_{1}\right)_{s}=n\left(\Gamma_{1 s}\right)$.

(b) If $\Gamma_{1}$ is saturated, then for any $\delta \in \Gamma, \bigcap_{n=-\infty}^{\infty} \operatorname{Ad} \delta^{n}\left(\Gamma_{1}\right)=$ $\bigcap_{i=1}^{k-1} \operatorname{Ad}^{*} \delta^{n_{i}}\left(\Gamma_{1}\right)$ for any $(k-1)$ values of $n_{i}$, when $\Gamma$ is $k$-step nilpotent.

(c) If $\delta_{1}, \delta_{2} \in \Gamma,\left(\delta_{1}, \delta_{2}\right)=0$ if and only if $\left(\delta_{1}^{n}, \delta_{2}^{m}\right)=0$ for some $m, n \neq 0$.

\section{Proof. C. H.}

Now let $I$ be a primitive ideal of $C^{*}(\Gamma)$, and let $U$ be an irreducible representation of $\Gamma$ with kernel $I$. Let $K(I)$ be the kernel of $U$ in $\Gamma$, and let $C(I)$ be the inverse image in $\Gamma$ of the center of $U$. Then $U_{\mid C(I)}$ is a one dimensional character $\chi(I) . K(I)$ is the subset of $\delta \in C(I)$ for which $\chi(I)(\delta)=1$. $C(I)$ is the subset of $\delta \in \Gamma$, such that all $f \in I^{\perp}$ are eigenfunctions under translation by $\delta$, with eigenvalue $\chi(I)(\delta)$. In particular $K$ and $C$ depend only on $I$. $K(I)_{s}$ is the subset of $\delta \in C(I)$ such that $\chi(I)(\delta)^{n}=1$ for some $n$. Clearly $K(I), C(I)$, etc. are normal subgroups of $\Gamma$. If $\pi: \Gamma \rightarrow \Gamma / K(I)_{s}$ is the natural map, then $\pi^{-1}(\mathscr{\digamma}(\pi(\Gamma)))=C(I)_{s}$. We write also $K(I)=$ $K\left(I^{\perp}\right)$ and so forth.

It is our purpose in this section to reduce $I^{\perp}$ to subgroups of $\Gamma$, eventually to $C(I)_{s}$.

Lemma 10. Notations as above. $C\left(I^{\perp}\right)_{s}$ is the largest of those subgroups $H$ such that $r_{H}\left(I^{\perp}\right)$ is finite dimensional. If $I^{\perp}$ is not finite dimensional, then choose $\delta \in \Gamma$ such that $\pi(\delta) \in \mathcal{Z}^{(2)}(\pi(\Gamma))$ $\pi\left(C(I)_{s}\right)$. Let $D(\delta)$ be the inverse image in $\Gamma$ of the centralizer of $\pi(\delta)$ in $\pi(\Gamma)$. Then $I^{\perp}$ can be reduced to $D(\delta)$.

Proof. Let $\left\{\delta_{i}\right\}_{i=1}^{n}$ be a set of coset representatives for $C(I)$ in $C(I)_{s}$. Then $\left\{U\left(\delta_{i}\right)\right\}$ span $U\left(l_{1}\left(C(I)_{s}\right)\right)$, so $I_{\mid C(I)_{s}}^{\perp}$ certainly is finite dimensional. Let $H_{0}$ be maximal among subgroups $H$ such that $r_{H}\left(I^{\perp}\right)$ is finite dimensional. Then clearly $H_{0}$ is saturated, and as $H_{0} \supseteqq C(I)$, 
$H_{0} \supseteq C(I)_{s}$. If $H_{0} \neq C(I)_{s}$, then $H_{0}$ contains $F$, a normal subgroup of $\Gamma$, strictly containing $C(I)_{s}$. As $r_{F}\left(I^{\perp}\right)$ is a fortiori finite dimensional, $U_{\mid F}$ is a sum of finitely many distinct finite dimensional representations which are permuted by $\mathrm{Ad}^{*} \Gamma / F$. Using Proposition 1, we see that there is $F^{\prime}$, an e.e. characteristic subgroup of $F$, such that $U_{\mid F^{\prime}}$ is a sum of finitely many distinct one dimensional representations, again permuted by $\mathrm{Ad}^{*} \Gamma / F^{\prime}$. By C. H., analogously with Lemma 1, we see $\left(\Gamma, F^{\prime}\right) \leqq K(I)_{s}$. From the discussion just before this lemma, this shows $F^{\prime} \subseteq C(I)_{s}$, which is a contradiction, establishing our first assertion.

Choose $\delta$ as stated. C. H. shows that there is $m \in \boldsymbol{Z}$ such that for all $a \in Z,\left(\delta^{a m}, \Gamma\right) \subseteq C(I)$, and $\left(\delta^{a m}, D(\delta)\right) \leqq K(I)$ for all $a$. By Lemma $9, D\left(\delta^{m}\right)=D(\delta)$, so assume $m=1$. Now if $y \notin D(\delta)$, $\chi(I)((\delta, y))^{a} \neq 1$ for any $a \in Z$. Define operators $T_{n}$ on $l_{\infty}(\Gamma)$ defined by $T_{n}(f)=1 /(n+1) \sum_{j=0}^{n} \mathrm{Ad}^{*} \delta^{j}(f)$. Clearly $T_{n}$ preserves $I^{\perp}$. Moreover, for $f \in I^{\perp}, y \in \Gamma, T_{n}(f)(y)=\left(1 /(n+1) \sum_{j=0}^{n} \chi((\delta, y))^{-j}\right) f(y)$. Thus, as $n$ goes to $\infty, T_{n} f$ approaches $\left(f_{\mid D(\delta)}\right)$ as a weak* limit, so we see $I^{\perp}$ is indeed reducible to $D(\delta)$.

Let us denote the process of reduction described above by $R\left(I^{\perp}, \Gamma, \delta, \Gamma_{1}\right)$, where $\Gamma_{1}=D(\delta)$ is the group to which we reduce $I^{\perp}$. Suppose we apply $R\left(I^{\perp}, \Gamma, \delta, \Gamma_{1}\right)$ to $I^{\perp}$ to obtain $r_{\Gamma_{1}}\left(I^{\perp}\right)$. By Lemma 8, we may choose $I_{1}^{\perp}$, a dual ideal of $\Gamma_{1}$, such that $r_{\Gamma_{1}}\left(I^{\perp}\right)$ is the $\operatorname{Ad}^{*} \Gamma / \Gamma_{1}$ quasiorbit generated by $I_{1}^{\perp}$, so $I^{\perp}=i\left(I_{1}^{\perp}\right)$. If $l_{1}^{\perp}$ is not finite dimensional, we may go through another reduction process $R\left(I_{1}^{\perp}, \Gamma_{1}, \delta_{2}, \Gamma_{2}\right)$, and so forth. Clearly this process must terminate in a finite dimensional dual ideal $I_{l}^{\llcorner}$on a saturated subgroup $\Gamma_{l}$ of $\Gamma$, and we will have $i\left(I_{l}^{\lrcorner}\right)=I^{\perp}$. Hence any primitive dual ideal is generated by a finite dimensional dual ideal on a subgroup. Moreover, we have

Proposition 2. Notations as above, let i be a one dimensional representation of $H \cong \Gamma_{l}$ such that $\psi$ induces $I_{l}^{\perp}$ on $\Gamma_{l}$. Then the representation $U^{\Downarrow, H}$ of $\Gamma$ is irreducible, with kernel $I^{\perp}$.

Proof. That $U^{\psi, H}$ has kernel $I^{\perp}$ follows from Lemma 7 . To show it is irreducible we invoke a theorem of Mackey ([10], p. 141). If $y \in \Gamma$, and $H \cap y^{-1} H y$ has finite index in $H$, then $y$ normalizes $\Gamma_{l}$. If $y \in \Gamma_{l}$, then $\mathrm{Ad}^{*} y \dot{\psi}$ and $\dot{\psi}$ are inequivalent on $H \cap y^{-1} H y$, since $\psi$ on $H$ induces $I_{l}^{\perp}$ by assumption. If $y \in n\left(\Gamma_{l}\right)-\Gamma_{l}$, then $y$ was eliminated at some stage $R\left(I_{i}^{\perp}, \Gamma_{i}, \delta_{i+1}, \Gamma_{i+1}\right)$. Now clearly $\delta_{j} \in C\left(I_{j}^{\perp}\right)$, and $C\left(I_{j}^{\perp}\right) \subseteq H$, and $\psi$ agrees with $\chi\left(I_{j}^{\perp}\right)$ on $C\left(I_{j}^{\perp}\right)$ for all $j \leqq l$. The fact that $y$ was eliminated by $\delta_{i+1}$ implies that $\left(\delta_{i+1}, y\right) \in$ $C\left(I_{i}^{\perp}\right)$ and $\chi\left(I_{i}^{\perp}\right)\left(\left(\delta_{i+1}, y\right)\right) \neq 1$. This implies that $\delta_{i+1} \in H \cap y^{-1} H y$, and $\operatorname{Ad}^{*} y \psi\left(\delta_{i+1}\right) \neq \psi\left(\delta_{i+1}\right)$. Hence the criteria of Mackey's result are 
satisfied, so $U^{\psi, H}$ is irreducible.

Thus the classical fact persists, slightly changed.

Lemma 11. $I^{\perp}$ can be reduced to $C\left(I^{\perp}\right)_{s}$.

Proof. Using $R(\quad)$ successively, reduce $I^{\perp}$ to the finite dimensional dual ideal $I_{l}^{\perp}$ on $\Gamma_{l}$. Then $K\left(I_{l}^{\perp}\right)_{s} \supseteqq \Gamma_{l}^{(2)}$. Take $\delta \in \Gamma$, and put $H=\bigcap_{n=-\infty}^{\infty} \operatorname{Ad} \delta^{n}\left(\Gamma_{l}\right)$, and let $H_{1}$ be the set of $h \in H$, such that $\left(\delta^{a}, h^{b}\right) \in H \cap K\left(I_{l}^{\perp}\right)_{s}$ for all $a, b \in Z$. Then C. H. shows that $H$ and $H_{1}$ are saturated subgroups of $\Gamma$, normalized by $\delta$, and $H_{1} \supseteqq H^{(2)}$. Again using C. H., it may be verified that for some $m>0,\left(\delta^{a m}, H\right) \in$ $C\left(I_{l}^{\perp}\right) \cap H$ and $\left(\delta^{a m}, H_{1}\right) \subseteq K\left(I_{l}^{\perp}\right) \cap H_{1}$ for all $a \in \boldsymbol{Z}$. As replacing $\delta$ by $\delta^{m}$ does not change $H$ or $H_{1}$, once more by C. H., we may assume $m=1$.

Now consider, for this $\delta$, the operators $T_{n}$, defined in Lemma 10. By the above, $\operatorname{Ad}^{*} \delta^{a} \dot{f}(h)=\dot{f}(h) \quad \chi\left(I_{l}^{\perp}\right)\left(\left(\delta^{a}, h\right)\right)$ for $h \in H$, and $f \in I_{l}^{\perp}$. Hence, if $h \in H_{1}, T_{n} f(h)=f(h)$. Also, if $y \in \Gamma-H$, Lemma 9 (b) implies $T_{n} f(y) \rightarrow 0$ as $n \rightarrow \infty$. Take $h \in H-H_{1}$. Then $T_{n} f(h)=$ $f(h)\left(1 /(n+1) \sum_{j=0}^{n} \chi\left(I_{l}^{\perp}\right)\left(\left(\delta^{j}, h\right)\right)\right.$. C. H. shows that this last summation has the form $\sum_{j=0}^{n} e^{i P(j)}$, where $P(j)$ is a polynomial. Moreover, by definition of $H_{1}, h \notin H_{1}$ implies at least one coefficient of $P$ is irrational. By a classical theorem of Weyl [15], this means $T_{n} f(h) \rightarrow 0$, as $n \rightarrow \infty$. Therefore, we can reduce $I^{\perp}$ to $H_{1}$. Repeating this process using other $\delta$ 's, we see we may reduce $I^{\perp}$ to a finite dimensional dual ideal $J^{\perp}$ on a saturated normal subgroup $F$ of $\Gamma$. Moreover, the fact that $\mathrm{Ad}^{*} \delta \chi\left(I_{l}^{\perp}\right)(h)=\chi\left(I_{l}^{\perp}\right)(h)$ for $h \in K\left(I_{l}^{\perp}\right) \cap H$, will imply that $\operatorname{Ad}^{*} \Gamma\left(r_{F}\left(\chi\left(I_{l}^{\perp}\right)\right)\right)$ is a finite set. Therefore, $r_{F}\left(I^{\perp}\right)$ is finite dimensional. Therefore, by Lemma $10, F \cong C\left(I^{\perp}\right)_{s}$. But clearly $I^{\perp}$ cannot be reduced to a saturated subgroup smaller than $C\left(I^{\perp}\right)_{s}$. Therefore $F=C\left(I^{\perp}\right)_{s}$.

CoROllary. Every primitive ideal in $C^{*}(\Gamma)$ is determined by its intersection with $l_{1}(\Gamma)$.

Proof. This is the same as to say, that if $I_{1}^{\perp} \neq I_{2}^{\perp}$ are two distinct primitive dual ideals of $\Gamma$, then the $w^{*}$-closed subspaces $M_{1}, M_{2}$, of $l_{\infty}(\Gamma)$ generated by $I_{1}^{\perp}, I_{2}^{\perp}$, are also distinct. But if $M_{1}=$ $M_{2}$, then, as every function in $M_{i}$ transforms by $\chi\left(I_{i}^{\perp}\right)$ under translation by elements of $C\left(I_{i}^{\perp}\right)$, we must have $C=C\left(I_{1}^{\perp}\right)=C\left(I_{2}^{\perp}\right)$, and $\chi\left(I_{1}^{\perp}\right)=\chi\left(I_{2}^{\perp}\right)$, and, also, of course $M_{1 \mid C_{s}}=M_{2 \mid C_{s}}$. But for finite dimensional dual ideals, the result is quite clear. Hence $I_{1 \mid C_{s}}^{\perp}=I_{2 \mid C_{s}}^{\perp}$. But now Lemma 11 shows $I_{1}^{\perp}=I_{2}^{\perp}$.

This corollary may be regarded as a sort of Tauberian theorem for $\Gamma$. 
By a trace on $\Gamma$, we understand a positive definite function $f$, constant on conjugacy classes, and satisfying $f(e)=1$ (e is the identity). See [14].

Proposition 3. Every primitive dual ideal $I^{\perp}$ contains exactly one trace, $\theta\left(I^{\perp}\right)$. If $I^{\perp}$ is finite dimensional, let $U$ be an irreducible representation of $\Gamma_{1}$ corresponding to $I^{\perp}$. Then if $\varphi(U)$ is the character of $U, \theta\left(I^{\perp}\right)=(\operatorname{dim} U)^{-1} \varphi(U)$. In general, let $\left\{J_{i}^{\perp}\right\}_{i=1}^{n}$ be the primitive dual ideals of $C\left(I^{\perp}\right)_{s}$ contained in $I^{\perp}$. Then $\theta\left(I^{\perp}\right)=$ $(1 / n) \sum_{i=1}^{n} \dot{\theta}\left(J_{i}^{\perp}\right)$.

Proof. The formula above clearly gives a trace contained in $I^{\perp}$. Let $\varphi$ be any trace in $I^{\perp}$. Then clearly $\varphi$ agrees with $\theta\left(I^{\perp}\right)$ on $C\left(I^{\perp}\right)_{s}$. Let notations be as in Lemma 10. Then the method of reduction in that lemma shows immediately that $\phi$ is zero on $\pi^{-1}\left(\mathscr{Z}^{(2)}\left(\Gamma / K\left(I^{\perp}\right)_{s}\right)\right)-C\left(I^{\perp}\right)_{s}$. We will show by induction on $i$, that $\varphi$ is zero on $H_{i}=\pi^{-1}\left(\mathscr{Z}^{(i)}\left(\Gamma / K\left(I^{\perp}\right)_{s}\right)\right)-C\left(I^{\perp}\right)_{s}$. Suppose it so for $i$, and take $\delta \in H_{i+1}-H_{i}$. Let $Y$ be the conjugacy class of $\delta$. Since $\left(\Gamma, H_{i+1}\right) \subseteq H_{i}$, every $g \in Y$ can be written $g=\delta \cdot h$, with $h \in H_{i}$. $\pi(y)$ is infinite. Therefore, either (1) for some $g_{0}=\delta h_{0}$, there are an infinite number of $g_{i}=\delta h_{i}$ such that $h_{0}^{-1} h_{i} \in C\left(I^{\perp}\right)_{s}-K\left(I^{\perp}\right)_{s}$; or (2) there are an infinite number of $g_{i}=\delta h_{i}$, such that $h_{i} h_{j}^{-1} \notin C\left(I^{\perp}\right)_{s}$, for $i \neq j$. If (1) holds, then, for some $i, h_{0}^{-1} h_{i} \in C\left(I^{\perp}\right)-K\left(I^{\perp}\right)$, so $\phi\left(g_{i}\right)=$ $\chi\left(h_{0}^{-1} h_{i}\right) \varphi\left(g_{0}\right)$, which is impossible unless $\varphi\left(g_{i}\right)=\varphi\left(g_{0}\right)=0$. If (2) holds, consider the element $a$ of $l_{1}(\Gamma)$ given by $a=e-\overline{\phi(\delta)}\left(\sum_{i=0}^{n} g_{i}\right)$.

Then $a^{*}=e-\varphi(\delta)\left(\sum_{i=0}^{n} g_{i}^{-1}\right)$, and $a^{*} a=e\left(1+n|\varphi(\delta)|^{2}\right)-\overline{\phi(\delta)}\left(\sum_{i=0}^{n} g_{i}\right)-$ $\varphi(\delta)\left(\sum_{i=0}^{n} g_{i}^{-1}\right)+|\varphi(\delta)|^{2}\left(\sum_{i \neq j} h_{j}^{-1} h_{i}\right)$. Hence $0 \leqq \varphi\left(a^{*} a\right)=1-n|\varphi(\delta)|^{2}$, for any $n$. But this is absurd, unless $\varphi(\delta)=0$, so the proposition is established.

Corollary. $M(\Gamma)$ is $T_{1}$. That is, every primitive ideal of $\Gamma$ is maximal.

Proof. If $I_{1} \subseteq I_{2}$ are two primitive ideals, then $I_{1}^{\perp} \supseteqq I_{2}^{\perp}$, so $\theta\left(I_{2}^{\perp}\right) \subseteq I_{1}^{\perp}$, so $\theta\left(I_{2}^{\perp}\right)=\theta\left(I_{1}^{\perp}\right)$, so $I_{1}^{\perp}=I_{2}^{\perp}$.

As an example, we give the traces of the primitive ideals of $H$, the "discrete Heisenberg group," cited in the introduction. Clearly for any primitive ideal $I$ of $C^{*}(H)$, $\mathscr{Z}(H) \cong C(I)$. If $\chi(I)$ is irrational, i.e., faithful on $\mathscr{Z}(H)$, then $\mathscr{Z}(H)=C(I)$, and the reduction process shows $\theta(I)$ is $\chi(I)$ on $\mathscr{Z}(H)$ and zero elsewhere.

If $\chi(I)$ is rational, however, $\#(\mathscr{Z}(H) / \operatorname{ker} \chi(I) \cap \mathscr{Z}(H))=n$ is finite. Let $H^{\prime}=H / \operatorname{ker} \chi(I) \cap \mathscr{Z}(H)$, and let $\pi: H \rightarrow H^{\prime}$ be the quotient map. Then $\sharp\left(H^{\prime} / \mathscr{Z}\left(H^{\prime}\right)\right)=n^{2}$ is also finite, and $\chi(I)$ is actually defined on $\pi^{-1}\left(\mathscr{Z}\left(H^{\prime}\right)\right)=C(I)$. It is an arbitrary extension of $\chi(I)$ 
on $\mathscr{Z}(H)$ to $C(I)$. An easy calculation shows that again $\Phi(I)$ is $\chi(I)$ on $C(I)$, and zero elsewhere. These facts, together with the description of the topology to be provided by Theorem 2, suffice to verify the description of $M(H)$ given earlier.

To finish this section, we note a result on type. We will say a primitive ideal $I$ of $C^{*}(\Gamma)$ is type one if there is only one irreducible representation of $\Gamma$ with kernel $I$. This is more or less consistent with existing terminology.

Proposition 4. If $I \leqq C^{*}(\Gamma)$ is a type one primitive ideal, $I^{\perp}$ is finite dimensional.

Proof. If $I^{\perp}$ is not finite dimensional, we can choose $\delta$ as in Lemma 10, and apply $R\left(I^{\lrcorner}, \Gamma, \delta, D(\delta)\right)$. Since $I^{\llcorner}$is minimal, any dual ideal $J^{\perp}$ of $D(\delta)$ contained in $r\left(I^{\perp}\right)$ will generate $I^{\perp}$. Since $\delta \in C\left(J^{\perp}\right)$, regardless of the $J^{\perp}$ chosen, choosing $J^{\perp}$ involves specifying $\chi\left(J^{\perp}\right)(\delta)$. It is easily seen that the spectrum of $U(\delta)$, where $U$ is any irreducible representation of $\Gamma$ with kernel $I$, is the whole unit circle. Hence $\chi\left(J^{\perp}\right)(\delta)$ may be specified arbitrarily. Let $H$ be the group generated by $\delta$ and $C\left(I^{\perp}\right)$. Then $H$ is normal, and $\mathrm{Ad}^{*} \Gamma / H$ acts on the one dimensional representations of $H$. Since $\Gamma$ is countable, there are an uncountable number of orbits. Pick $J_{1}^{\perp}, J_{2}^{\perp}$, so that $r_{H}\left(\chi\left(J_{1}^{\perp}\right)\right)$ and $r_{H}\left(\chi\left(J_{2}^{\perp}\right)\right)$ are in different orbits. Then, continuing the reduction of $J_{i}^{\perp}$, we will arrive at one dimensional characters on subgroups and then induce back up to get representations $U_{1}, U_{2}$. These representations are clearly inequivalent, for in each of them, $U_{i}(\delta)$ has pure point spectrum, and the points of the spectrum are different, by choice of $J_{1}^{\perp}, J_{2}^{\perp}$.

V. Classification of ideals. Here we return to the notations of $\S$ II. In particular, $\Gamma$ is now e.e. $L=\log \Gamma . \quad L^{\prime}=2 L . \quad \Gamma^{\prime}=$ $\exp L^{\prime}$. We shall analyze the structure of $\Delta\left(\Gamma^{\prime}, \Gamma\right)$ along lines parallel to the last section.

Consider 0 , an $\operatorname{Ad}^{*} \Gamma$ quasiorbit in $\hat{L}^{\prime}$. We let $\log K(0)$ be the sublattice of $L^{\prime}$, of elements $y$ such that $\psi(y)=1$ for all $\psi \in 0$. $\log C(0)$ is the set of $y$ such that the set $\{\psi(y): \psi \in 0\}$ is one number. Then it is not hard to verify (using Theorem 1) that $K(0)=$ $\exp (\log K(0))$ is a normal subgroup of $\Gamma^{\prime}$, as is $C(0)=\exp (\log C(0))$. $K(0)_{s}$ is seen to be the maximal subgroup of $\Gamma^{\prime}$ on which all $\psi \in 0$ are rational, and $C(0)_{s}$ is the largest of the groups $H$ such that $r_{H}(0)$ is finite. The common restriction of $\psi \in 0$ to $C(0)$ is a one dimensional character, which we will denote $\chi(0)$. Using C. H., it follows that if $\pi: \Gamma^{\prime} \rightarrow \Gamma^{\prime} / K(0)_{s}$ is the natural projection, then $C(0)_{s}=$ $\pi^{-1}\left(\mathscr{Z}\left(\pi\left(\Gamma^{\prime}\right)\right)\right)$. 
Lemma 12. Notations as above. If $C(0)_{s} \neq \Gamma^{\prime}$, then pick $\delta \in \Gamma^{\prime}$ such that $\pi(\delta) \in \mathscr{Z}^{(2)}\left(\pi\left(\Gamma^{\prime}\right)\right)-\pi\left(C(0)_{s}\right)$. Let $D^{\prime}=D^{\prime}(\delta)=\pi^{-1}(C(\delta))$, where $C(\delta)$ is the centralizer of $\pi(\delta)$ in $\pi\left(\Gamma^{\prime}\right)$. Put $\Delta^{\prime}=\log D^{\prime}$. Then $0=r^{-1}(r(0))$, where $r=r_{\Delta^{\prime}}$.

Proof. C. H. shows that for some $m>0, \operatorname{Ad}^{*} \delta^{m}(r(\psi))=r(\psi)$ for every $\psi \in 0$, and $\left(\delta^{a m}, D^{\prime}(\delta)\right) \subseteq C(0)$, and $\left(\delta^{a m}, D^{\prime}(\delta)\right) \subseteq K(0)$ for every $a \in Z$. As Lemma 9 guarantees $D^{\prime}(\delta)=D^{\prime}\left(\delta^{m}\right)$, assume $m=1$. Then C. H. gives, for $y \in L^{\prime}, \psi \in 0, \operatorname{Ad}^{*} \delta^{a}(\psi(y))=\psi(y) \psi(-n[\log \delta, y])$. Hence the set $S=\left\{\bar{\psi} \mathrm{Ad}^{*} \delta^{a}(\psi)\right\}$ forms a group of characters of $A=L^{\prime} / \Delta^{\prime}$. Since, for $y \notin \Delta^{\prime}, \operatorname{Ad}^{*} \delta^{a}(\psi)(y) \neq \psi(y)$ for any $a \in Z, \psi \in 0$, $S$ is dense in $A$. Hence $\left\{\operatorname{Ad}^{*} \delta^{n}(\psi)\right\}$ is dense in $r^{-1}(\psi)$. Letting $\psi$ range over 0 , the result follows.

Call the process described above $R\left(0, \Gamma^{\prime}, \delta, \Gamma_{1}^{\prime}\right)$, where $\Gamma_{1}^{\prime}=D^{\prime}$. Put $\Delta=(1 / 2) \Delta^{\prime}, D=D(\delta)=\exp \Delta$. We can pick an $\operatorname{Ad}^{*} D(\delta)$ quasiorbit $0_{1}$ in $\Delta^{\prime}$ whose $\mathrm{Ad}^{*} \Gamma$ quasiorbit is $r(0)$. Then we may repeat the reduction process, until we arrive at $\Gamma_{l}^{\prime}$, a saturated subgroup of $\Gamma^{\prime}$, and $0_{l}$, a finite $\mathrm{Ad}^{*} \Gamma_{l}$ quasiorbit in $\log \Gamma_{l}^{\prime}$, where $\Gamma_{l}=$ $\exp \left((1 / 2) \log \Gamma_{l}\right)$. Then an induction using Lemma 12 shows, if $\psi \in \hat{L}^{\prime}$, and $r_{l}(\psi) \in 0_{l}$, then $\mathrm{Ad}^{*} \Gamma_{l}(\psi)$ is dense in $r_{l}^{-1}(0)$.

Lemma 13. Let $V$ be an irreducible representation of $\Gamma_{l}^{\prime}$, associated to $0_{l}$. Then the induced representation $U^{V}$ of $\Gamma^{\prime}$ is irreducible.

Proof. $V$ is induced from a one dimensional character on a subgroup $H \subseteq \Gamma_{l}^{\prime}$. An argument entirely parallel to that of Proposition 2 , applied to $H$ and $\psi$, establishes the lemma.

We are now ready to prove the main result. We remark that the analysis of $M(\Gamma)$ in $\S I V$ can be trivially modified to apply to $M\left(\Gamma^{\prime}, \Gamma\right)$. We define $\theta(0)=\#\left(r_{C}(0)\right)^{-1} \sum_{\varphi \in r_{C}(0)} \dot{\varphi}$, where $C=C(0)$.

Theorem 2. Notations as above. There is a canonical homeomorphism $\alpha: \Delta\left(\Gamma^{\prime}, \Gamma\right) \rightarrow M\left(\Gamma^{\prime}, \Gamma\right)$. Canonical means, for any automorphism of $\Gamma$, the induced maps on the two spaces commute with $\alpha$. If $\alpha(0)=I$, then $C(0)=C(I), \chi(0)=\chi(I)$, and $\theta(0)=\theta(I)$.

Proof. Take $0 \in \Delta\left(\Gamma^{\prime}, \Gamma\right)$. Via Lemma 12, we can reduce 0 to a finite $\mathrm{Ad}^{*} \Gamma_{l}$ orbit $0_{l}$ on some subgroup $\Gamma_{l}^{\prime}$ of $\Gamma$. By Theorem 1, $0_{l}$ corresponds to a finite dimensional dual ideal $I_{l}^{\perp}$ of $\Gamma_{l}^{\prime}$, which defines a point of $M\left(\Gamma_{l}^{\prime}, \Gamma_{l}\right)$. Lemma 13 shows that $I_{l}^{\perp}$ generates a dual ideal $I^{\perp}$ of $\Gamma^{\prime}$, which defines a point of $M\left(\Gamma^{\prime}, \Gamma\right)$. We write $\alpha(0)=I^{\perp}$. Considering all possible reductions of 0 , we get a canonical relation $\alpha$. We shall show $\alpha$ is a bijection. 
First, we check that $C(0)=C(I), \chi(0)=\chi(I)$, and $\theta(0)=\theta(I)$. It is clear that any $I^{\perp} \in \alpha(0)$ is a subset of the positive functionals associated to $U^{\chi(0), C(0)}$, and as $\chi(0)$ is invariant under $\operatorname{Ad}^{*} \Gamma \mid C(0)$, all $f \in I^{\perp}$ by transform $\chi(0)$ under translation by $C(0)$. Therefore $C(0) \leqq C\left(I^{\perp}\right)$, and $\chi(0)$ and $\chi(I)$ agree on $C(0)$. On the other hand, $I_{\mid C\left(I^{\perp}\right)_{s}}^{\perp}$ is finite dimensional. If $I^{\perp}$ is induced from $I_{l}^{\perp} \in M\left(\Gamma_{l}^{\prime}, \Gamma_{l}\right)$, then the characters associated to $I_{l}^{\perp}$ are in $I^{\perp}$, and they are finite sums of restrictions to $\Gamma_{l}^{\prime}$ of elements of 0 . Likewise, all conjugates by elements of $\Gamma$ of these characters are in $I^{\perp}$, and their restrictions to $C\left(I^{\perp}\right)_{s}$, which is normal, are the same sums of the conjugate elements of 0 , and every restriction of an element of 0 to $C\left(I^{\perp}\right)_{s}$ is involved in one of these sums. Since these sums can span only a finite dimensional space, the restrictions of elements of 0 to $C\left(I^{\perp}\right)_{s}$ can be only finite in number, since distinct restrictions are linearly independent. Hence $C\left(I^{\perp}\right)_{s} \cong C(0)_{s}$, by the discussion preceding Lemma 12. Hence $C\left(I^{\perp}\right)_{s}=C(0)_{s}$. It is now easy to see that $\theta(0) \in$ $I^{\perp}$, and, as $\theta(0)$ is clearly a trace on $\Gamma^{\prime}, \theta(0)=\theta(I)$. From this, the equality $C\left(I^{\perp}\right)=C(0)$ follows, since $C(0)$ is the subset of $\delta \in C(0)_{s}$, such that $|\theta(0)|=1$ and similarly for $C\left(I^{\perp}\right)$. That $K(I)=K(0)$ is now evident.

The above shows $\alpha$ is a function. It also shows that $\alpha$ is onto. For Theorem 1 guarantees that, given $I^{\perp}$, there is 0 , such that $\alpha(r(0))=r\left(I^{\perp}\right)$, where $r$ restriction to $C\left(I^{\perp}\right)_{s}$, and $\alpha$ here is the map $\alpha: \Delta\left(C\left(I^{\perp}\right)_{s}, \Gamma\right) \rightarrow M\left(C\left(I^{\perp}\right)_{s}, \Gamma\right)$. But then it is clear that $C(0)_{s} \supseteqq C\left(I^{\perp}\right)_{s}$, and $\theta(0)_{\mid C\left(I^{\perp}\right)_{s}}=\theta\left(I^{\perp}\right)$. From this, it follows, as in Proposition 3, that $\theta(0)=\theta\left(I^{\perp}\right)$, and so indeed $I^{\perp}=\alpha(0)$. Finally, we show $\alpha$ is one-to-one. We may assume the theorem true for groups of lower rank than $\Gamma$. Since $\alpha$ is canonical, it follows that if $H \subseteq \Gamma^{\prime}$ is any proper normal saturated subgroup then $\Delta(H, \Gamma)$ and $M(H, \Gamma)$ are homeomorphic. Now take $0_{1}, 0_{2} \in \Delta\left(\Gamma^{\prime}, \Gamma\right)$, and suppose $\alpha\left(0_{1}\right)=$ $\alpha\left(0_{2}\right)=I^{\perp}$. Then $\chi\left(0_{1}\right)=\chi\left(0_{2}\right)=\chi\left(I^{\perp}\right)$, and we may reduce $0_{1}, 0_{2}$ and $I^{\perp}$ to $D^{\prime}(\delta)$ for some one $\delta$. Here we conclude that $\alpha\left(r\left(0_{1}\right)\right)=$ $\alpha\left(r\left(0_{2}\right)\right)=r\left(I^{\perp}\right)$, and since $\alpha$ is a bijection on this level, $r\left(0_{1}\right)=r\left(0_{2}\right)$, and so $0_{1}=0_{2}$, by Lemma 12 .

Finally we must compare the topologies. To do this essentially involves refining previous considerations, particularly Proposition 3, and Lemma 12. Let $\left\{I_{i}^{\perp}\right\}_{i=1}^{\infty}$ be a sequence of points of $M\left(\Gamma^{\prime}, \Gamma\right)$. Let $K_{j}=\bigcap_{i=j}^{\infty} K\left(I_{i}^{\perp}\right)$, and $C_{j}=\bigcap_{i=j}^{\infty} C\left(I_{i}^{\perp}\right)$, and $K=\bigcup_{j=1}^{\infty} K_{j}$, and $C=$ $\mathrm{U}_{j=1}^{\infty} C_{j}$. Inducing on the rank of $C_{j}$, then on $\#\left(\left(C_{j}\right)_{s} / C_{j}\right)$, we see there is an $m$ such that $K=K_{m}$, and $C=C_{m}$. Then we see that $K_{\mathrm{s}}$ is the subgroup of $\Gamma$, consisting of elements $\delta$, such that there exists $n$, such that $U_{i}\left(\delta^{n}\right)=1$, for all $i \geqq m$, where $U_{i}$ is a representation attached to $I_{i}^{\perp}$. If $\pi: \Gamma \rightarrow \Gamma / K_{s}$ is the natural map, then 
$C_{s}=\pi^{-1}\left(\mathscr{C}\left(\Gamma / K_{s}\right)\right) . \quad C_{s}$ may also be described as the largest of the subgroup $H \subseteq \Gamma$, such that there exists $d$, such that $\operatorname{dim}\left(U_{i}\left(l_{1}(H)\right)\right) \leqq d$ for all $i \geqq m$. Suppose that for any subgroup $D \subseteq C$ of finite index, containing $K$, that $C \cap K\left(I_{j}^{\perp}\right) \leqq D$ for $i$ sufficiently large. Then I claim $\lim _{i \rightarrow \infty} \theta\left(I_{i}^{\perp}\right)(\delta)=0$ for $\delta \notin C_{s}$.

To show this, let $C_{s}=H_{1}=\pi^{-1}\left(\mathscr{F}\left(\Gamma / K_{s}\right)\right)$, and $H_{i}=\pi^{-1}\left(\mathscr{C}^{(i)}\left(\Gamma / K_{s}\right)\right)$. For $\delta \in H_{2}-H_{1}$, we can find $y \in \Gamma$ such that $(y, \delta) \in C-K$. From now on, write $\chi\left(I_{i}^{\perp}\right)=\chi_{i}, \theta\left(I_{i}^{\perp}\right)=\theta_{i}$. Then, by our assumption above, for $i$ sufficiently large $\chi_{i}((y, \delta)) \neq 1$, so $\theta\left(I_{i}^{\perp}\right)(\delta)=0$. Now suppose $\delta \in H_{i+1}-H_{i}, i>1$. Now either there is $y \in \Gamma$, such that $(\delta, y) \in$ $C_{s}-K_{s}$, or, if $Y$ is the conjugacy class of $\delta$, then $\pi(Y)$ intersects each coset of $\pi\left(C_{s}\right)$ at most once. In the first case $\left(\delta, y^{a}\right) \in C-K$ for some $a$, and then $\theta_{i}(\delta)$ is eventually zero as before. Otherwise, we may pick an infinite sequence $\left\{g_{j}\right\}_{j=1}^{\infty}$ of elements of $Y$, such that, if $g_{j}=\delta h_{j}$, then $h_{i} h_{j}^{-1} \in H_{i}-H_{1}$. Construct $a$ as in Proposition 3, using $\theta_{l}$ instead of $\varphi$. Then we have $0 \leqq \theta_{l}\left(a^{*} a\right)=1+\left|\theta_{l}(\delta)\right|^{2}(-n+$ $\left.\sum_{i \neq j} \theta_{l}\left(h_{i}^{-1} h_{j}\right)\right)$. Choosing $l$ sufficiently large, we may conclude, by induction, that $\left|\theta_{l}\left(h_{i}^{-1} h_{j}\right)\right| \leqq 1 / 2 n^{2}$, so $\left|\theta_{l}(\delta)\right|^{2} \leqq 2 / n$. This establishes the claim.

Now suppose $S \subseteq M\left(\Gamma^{\prime}, \Gamma\right), I_{0}^{\perp} \in S$, and $\left\{I_{i}^{\perp}\right\}_{i=1}^{\infty} \cong S$ converges to $I_{0}^{\perp}$. Suppose $C$ and $K$ as constructed above are the same for all subsequences. (If $\left\{I_{i}^{\perp}\right\}_{i=1}^{\infty}$ does not satisfy this condition, we may find a subsequence which does.) Then $\chi\left(I_{i}^{\perp}\right)$ converges to $\chi\left(I_{0}^{\perp}\right)$ on $C$. Let $C_{1} \subseteq C$ be the subgroup on which $\chi\left(I_{i}^{\llcorner}\right)$is constant for $i$ sufficiently large. Then $K \subseteq C_{1}$, and conversely, $K$ contains $C_{1} \cap K\left(I_{0}^{\perp}\right)$. If $\delta \in C-C_{1}$, then $\chi\left(I_{i}^{\frac{1}{}}\right)$ is a nonconstant sequence converging to $\chi\left(I_{0}^{\perp}\right)(\delta)$ and so, by our assumption on $\left\{I_{i}^{\perp}\right\}_{i=1}^{\infty}, \chi\left(I_{i}^{\perp}\right)(\delta) \neq 1$, for $i$ large. It follows that the $\chi_{i}$ satisfy the condition discussed above, and so $\theta_{i}(\delta) \rightarrow 0$ for $\delta \notin C_{s}$. We conclude immediately that $\left\{I_{i}^{\perp}\right\}$ converges to all dual ideals $J^{\perp}$ such that $\theta\left(J^{\perp}\right)_{\mid C}=\theta\left(I_{0}^{\perp}\right)_{I C}$.

Now we notice that the topology of $\Delta\left(\Gamma^{\prime}, \Gamma\right)$ is given by the quotient of the weak *topology by the action of $\mathrm{Ad}^{*} \Gamma$ on the positive definite functions of $L^{\prime}=\log \Gamma^{\prime}$. Using this fact, we may repeat the above analysis, substituting $\theta\left(0_{i}\right)$ 's for $\theta\left(I_{i}^{\perp}\right)$ 's. Then we see the topologies are indeed the same by virtue of the identity of $\theta(0)$ and $\theta(\alpha(0))$.

This concludes Theorem 2.

REMARKs. (a) Perhaps the best way to think of the above analysis is to regard it as establishing the equality of the topologies of both $\Delta\left(\Gamma^{\prime}, \Gamma\right)$ and $M\left(\Gamma^{\prime}, \Gamma\right)$ with Effros' simplex topology [5] on the extremal traces of $\Gamma$, which are identified via exp with the $\operatorname{Ad}^{*} \Gamma$ invariant states on $L^{\prime}$.

(b) Theorem 2 implies that the orbits in $\Delta\left(\Gamma^{\prime}, \Gamma\right)$ have a fairly 
simple structure. 0 is a finite union of cosets of the subtorus of $L^{\prime}$ which annihilates $C(0)_{s}$.

(c) One way of looking at Theorem 2 is as a duality theorem. If $G$ is an extension of $\Gamma$ by $\log \Gamma$, for example, the semidirect product, and $G^{\prime}$, the included extension of $\Gamma^{\prime}$ by $\log \Gamma^{\prime}$, then Theorem 2 says $M\left(\log \Gamma^{\prime}, G\right)$ and $M\left(G^{\prime} / \log \Gamma^{\prime}, G\right)$ are naturally isomorphic. The significance of this interpretation is unclear.

VI. Application to finite groups, Let $\Gamma$ be e.e., and $\Gamma_{1} \subseteq \Gamma$ a normal e.e. subgroup. Put $L=\log \Gamma, L_{1}=\log \Gamma_{1}$. We will say $\Gamma_{1}$ is e.e. embedded if $\left[L, L_{1}\right] \subseteq k ! L_{1}$, where, as usual, $\Gamma$ is $k$-step nilpotent. If $\Gamma_{1} \subseteq \Gamma$ is e.e. embedded, C. H. shows immediately, that for $\delta \in \Gamma, \log \delta \Gamma_{1}=\log \delta+L_{1}$. Hence functions on $L$, constant on cosets of $L_{1}$, can be interpreted as functions on $\Gamma / \Gamma_{1}$. In particular, the annihilator of $L_{1}$ in $\hat{L}$ defines an orthogonal basis for $l_{2}\left(\Gamma / \Gamma_{1}\right)$. exp: $L \rightarrow \Gamma$ can also be factored to a map exp: $L / L_{1} \rightarrow \Gamma / \Gamma_{1}$.

LemMa 15. If $p$ is a prime, and $p>k$, and $\Gamma_{1} \subseteq \Gamma$ is normal, and $\sharp\left(\Gamma / \Gamma_{1}\right)=p^{m}, m \in Z$, then $\Gamma_{1}$ is e.e. embedded in $\Gamma$.

Proof. Let $L_{1}$ be the lattice generated by $\log \Gamma_{1}$. Then certainly $L_{1} \subseteq L=\log \Gamma$, and also $p^{m} L \subseteq \log \Gamma_{1} \subseteq L_{1}$. Also, Proposition 0 gives $k !^{2} L_{1} \subseteq \log \Gamma_{1}$. Therefore, as $p$ and $k$ ! are relatively prime by assumption, $L_{1}=\log \Gamma_{1}$. C. H. now shows $\left[L, L_{1}\right] \subseteq(k-1) !^{-1} L_{1}$, or $k !(k-1) !\left(k !^{-1}\left[L, L_{1}\right]\right) \subseteq L_{1}$. But as $k !^{-1}\left[L, L_{1}\right] \subseteq L$, we have also $p^{m}\left(k !^{-1}\left[L, L_{1}\right]\right) \subseteq L_{1}$. Hence $\left[L, L_{1}\right] \subseteq k ! L_{1}$. A fortiori $L_{1}$ is e.e. and so is e.e. embedded.

Now let $P$ be a $p$-group, which is $(p-1)$-step nilpotent. Then if $p_{1}, \cdots, p_{n}$ generate $P$, the natural map $\pi$ from $F$, the free group on $p_{1}, \cdots, p_{n}$, onto $P$, factors to a map $\pi$ on $\Gamma=F / F^{(p)}$, which is known [13], to be a discrete, finitely generated, torsion-free, $(p-1)$ step nilpotent group. Let $\Gamma^{\prime} \cong \Gamma$ be the kernel of $\pi$. By Proposition 0 , there is a normal e.e. subgroup $\Gamma_{1}$ of $\Gamma$, such that if $\delta \in \Gamma$, $\delta^{k !^{2}} \in \Gamma_{1}$. Now $\pi$ restricts to a map $\pi: \Gamma_{1} / \Gamma_{1} \cap \Gamma^{\prime} \rightarrow P$, which we see is onto, since $k !^{2}$ and $p$ are relatively prime. Therefore, Lemma 14 shows $H=\Gamma_{1} \cap \Gamma^{\prime}$ is e.e. embedded in $\Gamma_{1}$. Therefore, we have the following result.

TheOREM 3. Let $P$ be $a(p-1)$-step nilpotent p-group. Then there is an abelian group $L$, which also has the structure of a $(p-1)$-step nilpotent lie algebra, and a bijection exp: $L \rightarrow P$, satisfying the Campbell-Hausdorff formula. Inner automorphisms of $P$ induce an action by automorphisms of $P$ on $L$, denoted Ad. The dual action on $\hat{L}$ is denoted $\mathrm{Ad}^{*}$. Via exp, the elements of $\hat{L}$ may 
be viewed as a multiplicative group of functions on $P$, which are permuted by inner automorphism, and are an orthogonal basis for $l_{2}(P)$. The irreducible representations of $P$ are in one-to-one correspondence with the $\mathrm{Ad}^{*} P$ orbits in $\hat{L}$. If 0 and $U_{0}$ correspond, then $\operatorname{dim} U_{0}=\#(0)^{1 / 2}$. The character, $\xi_{u}$ of $U$ is given by $\xi_{u}=$ $\#(0)^{-1 / 2} \sum_{\varphi \in 0} \varphi$. $U_{0}$ may be realized as an induced representation as follows: take any $\psi \in 0$, and any $Q \subseteq P$, a subgroup such that $\psi_{1 Q}$ is a one dimensional character, and $\#(Q)$ is maximal with respect to this property. Then $U_{0}=U^{\psi, Q}$.

We have also these results on induced representations and tensor products.

CoRollary 1. If $P$ and $P^{\prime}$ are as in the theorem, $P^{\prime} \cong P, L^{\prime}$ and $L$, the associated groups, and $r: \hat{L} \rightarrow \hat{L}^{\prime}$ the natural map; and if $O^{\prime}$ is an orbit in $\hat{L}^{\prime}$, and 0 an orbit in $\hat{L}$; then the multiplicity of $U_{0}$ in the representation induced from $U_{0^{\prime}}$ is $\#\left(0 \cap r^{-1}\left(0^{\prime}\right)\right) \#\left(0^{\prime}\right)^{-1 / 2}$ $\#(0)^{-1 / 2}$.

If $0_{1}, 0_{2}$ are two orbits in $L$, then the multiplicity of orbit $0_{3}$ in $0_{1}+0_{2}$ is the number of distinct ways some given $\psi_{3} \in 0_{3}$ can be represented in the form $\psi_{3}=\psi_{1} \psi_{2}$, with $\psi_{i} \in 0_{i}$.

COROLlary 2. If $k$ is the multiplicity of $0_{3}$ in $0_{1} 0_{2}$, then the multiplicity of $U_{0_{3}}$ in $U_{0_{1}} \otimes U_{0_{2}}$ is $k \#\left(0_{3}\right)^{1 / 2} \#\left(0_{1}\right)^{-1 / 2} \#\left(0_{2}\right)^{-1 / 2}$.

\section{APPENDIX. Proposition 0.}

Proposition 0 essentially involves inverting the Campbell-Hausdorff formula. That $\left[\log \Gamma^{* *}, \log \Gamma^{* *}\right] \subseteq(k-1) !^{-1} \log \Gamma^{* *}$ is a simple calculation. The longer part is to show $k !^{2} \log \Gamma^{* *} \cong \log \Gamma$. We begin with two observations crucial to the purpose.

(1) We recall the definition of the order of a commutator in a lie algebra $N$. Any $x \in N$ is a first order commutator. The bracket of an $i$ th order with a $j$ th order commutator is an $i+j$ th order commutator. Then, if $N$ is $k$-step nilpotent, C. H. for $N$ takes the form $\log \left(\prod_{i=1}^{n} \exp x_{i}\right)=\sum_{j=0}^{k} j !^{-2} S_{j}$, where $S_{j}$ is an integral sum of $j$ th order commutators in the $x_{i}$ 's.

(2) If $C\left(x_{i}\right)$ is a commutator in the $x_{i}$ 's, then $C\left(\exp x_{i}\right)$, the parallel commutator in the exp $x_{i}$ 's is gotten from $C\left(x_{i}\right)$ by replacing $x_{i}$ by $\exp x_{i}$, and each bracket by a like oriented parenthesis. If $C\left(x_{i}\right)$ is of $j$ th order, then C. H. gives $\log C\left(\exp x_{i}\right)=C\left(x_{i}\right)+(1 / 2) S_{j+1}+$ $\sum_{l=j+2}^{k} l !^{-2} S_{l}$, where $S_{l}$ is again an integral sum of commutators in the $x_{i}$ 's. 
Now with $\Gamma$ as always, $\log \Gamma \leqq N$, we want to show $k !^{2} \Sigma x_{i} \epsilon$ $\log \Gamma$. We consider $\log \left(\prod_{i=1}^{n} \exp \alpha x_{i}\right)=\alpha \Sigma x_{i}+\sum_{j=1}^{k} \alpha^{j} j !^{-2} S_{j}$, and seek to modify it successively by elements from $\log \Gamma$ in order to eliminate the higher order terms. Suppose at some point in this process, we have an expression $E=\alpha \Sigma x_{i}+C \sum_{j=l}^{k} \beta^{j} j !^{-2} S_{j}$. Assume $C \beta^{l} l !^{-2}$ is integral and write $C \beta^{l} l !^{-2}=C^{\prime}{\beta^{\prime}}^{l}$, where $C^{\prime}$ is not divisible by $d^{l}$ for any $d \in Z$. Suppose $S_{l}=\Sigma r_{m} C_{m}\left(x_{i}\right)$, where the $C_{m}$ are commutators, and $r_{m}$ are integers. Then multiply $\exp E$ by $\Pi C_{m}\left(\exp \beta^{\prime} x_{i}\right)^{-C^{\prime} r_{m}}=\exp F$. Then C. H. shows that $\log (\exp E \exp F)=$ $E^{\prime}=\alpha \Sigma x_{i}+C^{\prime} \sum_{j=l+1}^{k} \beta^{\prime j} j !^{-2} T_{j}$, where the $T_{j}$ are new integral sums of $j$ th order commutators in the $x_{i}$ 's. The problem now becomes computing $\alpha$ so that this process may be carried through the $k$ th step. Factoring $\alpha$ into primes, we see we may compute the necessary power of each prime separately. First take $p>2$. If $l=$ $\sum_{l=0}^{g} a_{i} p^{i}$, then $p$ appears in $j$ ! to the power $\nu(l)=(l-r(l))(p-1)^{-1}$ times, where $r(l)=\sum_{i=0}^{g} a_{i}$. If, at the $l$ th step, the power of $p$ in $C \beta^{l}$ is $p^{b}\left(p^{d}\right)^{l}$, and $b<2 \nu(l)$, so that is necessary to withdraw a power of $p$ from $p^{d}$ in order to proceed, we will call this process "dropping an exponent." So suppose we are at the $l$ th place, with $p$ appearing to the power $b+d l-2 \nu(l)$ and we must drop an exponent. Doing so, we see from the form of $\nu(l)$, that we may proceed to the $l+((p-1) / 2)$ th the stage without dropping an exponent. There the power of $p$ will be $b+q^{-1}\left(\sum_{\substack{q=0 \\ i=0}}(r(l+i)-i)\right)+$ $(d-1)(l+q)-2 \nu(l+q)$, where $q=(p-1) / 2$. Here again we will probably (but not always) have to drop another exponent.

Let us start at $l=a p^{i}$, and proceed, dropping an exponent every $q$ th step, until we are come to $m=(a+1) p^{i}-1$. If our initial power of $p$ is $b+d a p^{i}-2 \nu\left(a p^{i}\right)$, we shall arrive to find the power $\sigma+m\left(d-q^{-1}\left(p^{i}-1\right)-2 \nu(m)\right)$, where $\sigma$, the accumulated surplus, is given by $\sigma=q^{-1}\left(S(m-1)-S(l-1)-\left(p^{i}-1\right)(q-1)\right)+b$. Here $S(n)=\sum_{i=1}^{n} r(i)$. If we calculate this, we find $\sigma>2 \nu(m)$, so we may proceed to $m+1$ without dropping an exponent, and then carry on. Beginning from zero, we see that we need at most $2 \nu(k)$ powers of $p$ to carry through the $k$ th step. For $p=2$, the same argument works if we drop two exponents at each step, except just before powers of 2. Putting this together, we see $\alpha=k !^{2}$ is indeed sufficient to begin with to carry through the whole procedure to the $k$ th step.

From this, the existence of $\Gamma_{1}$ as stated in Proposition 0 is immediate. To construct $\Gamma_{2}$ is independent of the argument above. Put $L_{i}=N^{(i)} \cap\left(\log \Gamma^{* *}\right)$, and $\log \Gamma_{2}=\sum_{i=0}^{k-1}(k !(k-1 !))^{-i} L_{i}$. This will work. 


\section{REFERENCES}

1. L. Auslander et al., Flows on homogeneous spaces, Ann. of Math. Studies, No. 53, Princeton Univ. Press, 1963.

2. L. Auslander and B. Kostant, Quantization and representations of solvable Lie groups, B.A.M.S., 73 (1967), 692-95.

3. M. P. Bernat, Sur les representations unitaires des groupes de Lie resolubles, Ann. Sci. Ecole Norm. Sup., 82 (1965), 37-99.

4. J. Brezin, Unitary representation theory for solvable Lie groups, Memoirs of Amer. Math. Soc., No. 79 (1968).

5. E. Effros and F. Hahn, Transformation groups and $C^{*}$-algebras, Memoirs of Amer. Math. Soc., No. 75 (1967).

6. J. M. G. Fell, The dual spaces of $C^{*}$-algebras, Trans. A.M.S., 94 (1960), 365-403.

7. J. Glimm, Families of induced representations, Pacific J. Math., 12 (1962), 885-911.

8. F. P. Greenleaf, Amenable actions of locally compact groups, J. Functional Analysis, 4 (1969), 295-315.

9. A. A. Kirillov, Unitary representations of nilpotent Lie groups, Uspekhi. Matem. Nauk., 106 (1962), 57-110.

10. G. W. Mackey, Theory of group representations, University of Chicago, mimeographed notes, 1955.

11. C. C. Moore, Decomposition of unitary representations defined by discrete subgroups of nilpotent groups, Ann. of Math., 82 (1965), 146-182.

12. R. T. Prosser, On the ideal structure of operator algebras, Memoirs of Amer. Math. Soc., No. 45 (1963).

13. J. P. Serre, Lie Algebras and Lie Groups, W. A. Benjamin, Inc., 1965.

14. E. Thoma, Über unitare Darstellungen abzahlbarer diskreter Gruppen, Math. Annalen, 153 (1964), 111-141.

15. H. Weyl, Über die Gleichverteilung von Zahlen mod. Eins, Math. Annalen, 77 (1916), 313-352.

Received May 21, 1977.

YALE UNIVERSITY

New Haven, CT 06520 



\title{
PACIFIC JOURNAL OF MATHEMATICS
}

\section{EDITORS}

\author{
RICHARD ARENS (Managing Editor) \\ University of California \\ Los Angeles, CA 90024 \\ Charles W. Curtis \\ University of Oregon \\ Eugene, OR 97403 \\ C. C. MOORE \\ University of California \\ Berkeley, CA 94720
}

\author{
J. DUGUNDJI \\ Department of Mathematics \\ University of Southern California \\ Los Angeles, CA 90007
}

R. Finn and J. Milgram

Stanford University

Stanford, CA 94305

\section{ASSOCIATE EDITORS}

E. F. BeCKenBaCH

B. H. NeumanN

F. WOLF

K. YoSHIDA

\section{SUPPORTING INSTITUTIONS}

\author{
UNIVERSITY OF BRITISH COLUMBIA \\ CALIFORNIA INSTITUTE OF TECHNOLOGY \\ UNIVERSITY OF CALIFORNIA \\ MONTANA STATE UNIVERSITY \\ UNIVERSITY OF NEVADA, RENO \\ NEW MEXICO STATE UNIVERSITY \\ OREGON STATE UNIVERSITY \\ UNIVERSITY OF OREGON
}

\author{
OSAKA UNIVERSITY \\ UNIVERSITY OF SOUTHERN CALIFORNIA \\ STANFORD UNIVERSITY \\ UNIVERSITY OF HAWAII \\ UNIVERSITY OF TOKYO \\ UNIVERSITY OF UTAH \\ WASHINGTON STATE UNIVERSITY \\ UNIVERSITY OF WASHINGTON
}

The Supporting Institutions listed above contribute to the cost of publication of this Journal, but they are not owners or publishers and have no responsibility for its content or policies.

Mathematical papers intended for publication in the Pacific Journal of Mathematics should be in typed form or offset-reproduced, (not dittoed), double spaced with large margins. Please do not use built up fractions in the text of the manuscript. However, you may use them in the displayed equations. Underline Greek letters in red, German in green, and script in blue. The first paragraph or two must be capable of being used separately as a synopsis of the entire paper. Items of the bibliography should not be cited there unless absolutely necessary, in which case they must be identified by author and journal, rather than by item number. Manuscripts, in triplicate, may be sent to any one of the editors. Please classify according to the scheme of Math. Reviews, Index to Vol. 39. All other communications should be addressed to the managing editor, or Elaine Barth, University of California, Los Angeles, California, 90024.

The Pacific Journal of Mathematics expects the author's institution to pay page charges, and reserves the right to delay publication for nonpayment of charges in case of financial emergency.

50 reprints to each author are provided free for each article, only if page charges have been substantially paid. Additional copies may be obtained at cost in multiples of 50 .

The Pacific Journal of Mathematics is issued monthly as of January 1966. Regular subscription rate: $\$ 72.00$ a year (6 Vols., 12 issues). Special rate: $\$ 36.00$ a year to individual members of supporting institutions.

Subscriptions, orders for numbers issued in the last three calendar years, and changes of address should be sent to Pacific Journal of Mathematics, 103 Highland Boulevard, Berkeley, California, 94708. Older back numbers obtainable from Kraus Periodicals Co., Route 100, Millwood, NY 10546.

\footnotetext{
PUBLISHED BY PACIFIC JOURNAL OF MATHEMATICS, A NON-PROFIT CORPORATION

Printed at Kokusai Bunken Insatsusha (International Academic Printing Co., Ltd.). 8-8, 3-chome, Takadanobaba, Shinjuku-ku, Tokyo 160, Japan.
} 


\section{Pacific Journal of Mathematics}

\section{Vol. 73, No. $2 \quad$ April, 1977}

Roger Evans Howe, On representations of discrete, finitely generated, torsion-free, nilpotent groups ........................ 281

Roger Evans Howe, The Fourier transform for nilpotent locally compact

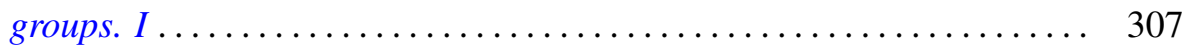

Roger Evans Howe, On a connection between nilpotent groups and

oscillatory integrals associated to singularities............... 329

Roger Evans Howe, Kirillov theory for compact p-adic groups .......... 365

Roger Evans Howe, Topics in harmonic analysis on solvable algebraic

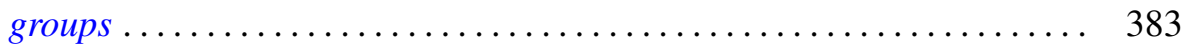

Roger Evans Howe, Tamely ramified supercuspidal representations of

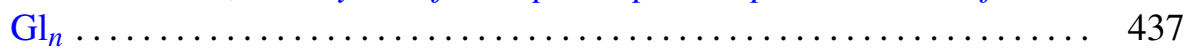

Lawrence Jay Corwin and Roger Evans Howe, Computing characters of tamely ramified $p$-adic division algebras .....................

Roger Evans Howe, Some qualitative results on the representation theory of

$\mathrm{Gl}_{n}$ over a $p$-adic field ............................. 479

Herbert Stanley Bear, Jr., Corrections to: “Ordered Gleason parts”. . . . . . 539

Andreas Blass, Corrections to: "Exact functors and measurable cardinals" .....................................

Robert M. DeVos, Corrections to: "Subsequences and rearrangements of sequences in $\mathrm{FK}$ spaces". 